\title{
AN OPTIMAL BINDING NUMBER CONDITION FOR BIPANCYCLISM*
}

\author{
ZHIQUAN $\mathrm{HU}^{\dagger}, \mathrm{KA}$ HO LAW ${ }^{\ddagger}$, AND WENAN ZANG§
}

\begin{abstract}
Let $G=\left(V_{1}, V_{2}, E\right)$ be a balanced bipartite graph with $2 n$ vertices. The bipartite binding number of $G$, denoted by $B(G)$, is defined to be $n$ if $G=K_{n, n}$ and

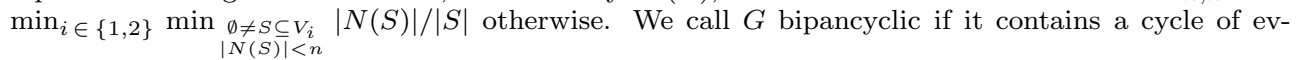
ery even length $m$ for $4 \leq m \leq 2 n$. The purpose of this paper is to show that if $B(G)>3 / 2$ and $n \geq 139$, then $G$ is bipancyclic; the bound $3 / 2$ is best possible in the sense that there exist infinitely many balanced bipartite graphs $G$ that have $B(G)=3 / 2$ but are not Hamiltonian.
\end{abstract}

Key words. bipartite graph, Hamiltonian cycle, bipancyclism, binding number

AMS subject classification. $05 \mathrm{C} 38$

DOI. $10.1137 / 120886443$

1. Introduction. Let $G=(V, E)$ be a graph. The binding number of $G$, denoted by $b(G)$, is defined to be

$$
\min _{\substack{\emptyset \neq S \subseteq V \\|N(S)|<|V|}}|N(S)| /|S|
$$

where $N(S)=\{v \in V: u v \in E$ for some $u \in S\}$. This parameter was introduced by Woodall [8] to measure how well the vertices of $G$ are bound together; in particular, if $b(G)$ is large, then $G$ has lots of edges fairly well distributed. The binding number resembles some other graph invariants, such as the minimum degree, connectivity, and toughness, in certain ways while providing more global structural information. In the literature there are a number of results showing that various properties of $G$ are consequences of assumptions on the value of $b(G)$, including the following theorem on Hamiltonian cycles.

TheOrem 1.1 (Woodall [8]). Every graph $G$ with $b(G) \geq 3 / 2$ is Hamiltonian.

Call $G$ pancyclic if it contains a cycle of every length $m$ for $3 \leq m \leq|V|$. As conjectured by Woodall [8] and proved by Shi [6,7], this assertion can be strengthened as follows.

TheOREm 1.2 (Shi $[6,7]$ ). Every graph $G$ with $b(G) \geq 3 / 2$ is pancyclic.

Observe that for bipartite graphs, the binding number does not give much information about their structures (or well-boundness) when compared to nonbipartite graphs. For instance, both $K_{n, n}$ (a complete bipartite graph) and $n K_{2}$ (union of $n$ disjoint edges) have binding number 1 for $n \geq 1$; their structures, however, are dramatically different. Furthermore, for any bipartite graph $G=\left(V_{1}, V_{2}, E\right)$, we have

${ }^{*}$ Received by the editors July 30, 2012; accepted for publication (in revised form) January 3, 2013; published electronically April 4, 2013.

http://www.siam.org/journals/sidma/27-2/88644.html

${ }^{\dagger}$ Faculty of Mathematics and Statistics, Central China Normal University, Wuhan, China (hu_zhiq@yahoo.com.cn). This author was supported in part by NSFC grants 11071096 and 11271149 and grant D20111110 of the Hubei Provincial Department of Education.

${ }^{\ddagger}$ Corresponding author. Department of Mathematics, University of Hong Kong, Hong Kong, China (lawkaho@graduate.hku.hk).

$\S$ Department of Mathematics, University of Hong Kong, Hong Kong, China (wzang@maths.hku. hk). This author was supported in part by the Research Grants Council of Hong Kong. 
$b(G) \leq \min \left\{\left|V_{2}\right| /\left|V_{1}\right|,\left|V_{1}\right| /\left|V_{2}\right|\right\} \leq 1$. Hence neither Theorem 1.1 nor Theorem 1.2 applies to $G$. In graph theory it is common for results to have a "bipartite" version; such a typical example is Jackson's theorem [3], which asserts that every 2-connected $k$-regular graph with at most $3 k$ vertices is Hamiltonian. Häggkvist [2] conjectured that every 2-connected $k$-regular bipartite graph $G$ with at most $6 k-6$ vertices is Hamiltonian, which was confirmed by Jackson and Li [4] when $G$ contains at most $6 k-38$ vertices. So a natural question to ask is, what are the counterparts of the above binding number theorems on bipartite graphs? To find the answer, clearly we need a new concept of binding number in order to better reflect the bipartiteness.

Let $G=\left(V_{1}, V_{2}, E\right)$ be a balanced bipartite graph with $2 n$ vertices. The bipartite binding number of $G$, denoted by $B(G)$, is defined to be $n$ if $G=K_{n, n}$ and

$$
\min _{i \in\{1,2\}} \min _{\substack{\phi \neq S \subseteq V_{i} \\|N(S)|<n}}|N(S)| /|S|
$$

otherwise. We call $G$ bipancyclic if it contains a cycle of every even length $m$ for $4 \leq m \leq 2 n$. The purpose of this paper is to establish the following bipartite version of the above two theorems.

THEOREM 1.3. Let $G$ be a balanced bipartite graph with $2 n$ vertices. If $B(G)>$ $3 / 2$ and $n \geq 139$, then $G$ is bipancyclic.

We shall exhibit infinitely many balanced bipartite graphs $G$ that have $B(G)=$ $3 / 2$ but are not Hamiltonian in section 2 (see Proposition 2.5). Thus the bound $3 / 2$ in our theorem is best possible. Moreover, the proof techniques of our theorem are substantially different from those of Theorems 1.1 and 1.2.

Let us introduce some notation before proceeding. Given a graph $G$, we use $V(G)$ and $E(G)$ to denote its vertex set and edge set, respectively. For each $v \in V(G)$, we use $d(v)$ and $N(v)$ to denote its degree and neighborhood, respectively. For each $S \subseteq V(G)$, it is clear that $N(S)=\cup_{v \in S} N(v)$. For each subgraph $H$ of $G$, let $G-H$ denote the subgraph of $G$ induced by $V(G)-V(H)$ and set $N_{H}(S):=N(S) \cap V(H)$. When $G$ is a bipartite graph with bipartition $\left(V_{1}, V_{2}\right)$, we set $V_{i}(H):=V_{i} \cap V(H)$ for $i=1,2$.

Throughout this paper, we use $C_{n}$ to denote a cycle of length $n$ and assume that each cycle $C$ has an implicit clockwise orientation. With this assumption, $v_{C}^{+}$and $v_{C}^{-}$ will stand for the successor and predecessor of a vertex $v$ on $C$ under this orientation, respectively; we shall drop the subscript $C$ if there is no danger of confusion. We define $v^{+i}$ recursively by $v^{+0}=v$ and $v^{+(i+1)}=\left(v^{+i}\right)^{+}$for $i \geq 0$ and define $v^{-i}$ analogously. For any two vertices $u$ and $v$ on $C$, let $u \vec{C} v$ denote the path from $u$ to $v$ on $C$ in the clockwise direction, and let $u \overleftarrow{C} v$ denote the path from $u$ to $v$ on $C$ in the counterclockwise direction. Set $C[u, v]:=V(u \overleftarrow{C} v)$ and $C(u, v]:=C[u, v]-\{u\}$ etc. For each $X \subseteq V(C)$ and $i \geq 1$, define $X^{+i}:=\left\{x^{+i}: x \in X\right\}$ and $X^{-i}:=$ $\left\{x^{-i}: x \in X\right\}$. If $X=N_{C}(v)$ for some vertex $v$, then we shall simply write $N_{C}^{+i}(v)$ and $N_{C}^{-i}(v)$ as opposed to the more cumbersome $\left(N_{C}(v)\right)^{+i}$ and $\left(N_{C}(v)\right)^{-i}$. We also define $X^{+0}:=X=: X^{-0}$ for convenience.

The remainder of this paper is organized as follows. In section 2 , we derive some basic properties satisfied by bipartite binding numbers. In section 3, we show the existence of certain nested cycle structures in $G$ under some assumptions. In section 4, we first establish a bipartite version of the hopping lemma originally developed by Woodall [8] and then employ it to further grow the nested cycle structures obtained in section 3 under some other assumptions. In section 5 , we prove that $G$ contains a cycle of every even length based on the aforementioned nested cycle structures. 
2. Preliminaries. Let $G=\left(V_{1}, V_{2}, E\right)$ be a balanced bipartite graph with $2 n$ vertices such that $G \neq K_{n, n}$. Recall the definition of the bipartite binding number $B(G)$; a subset $S$ of $V_{i}$, for $i=1$ or 2 , is called a binding set of $G$ if $|N(S)|<n$ and $B(G)=|N(S)| /|S|$.

The following proposition asserts that the value of $B(G)$ is uniquely determined by $G$ rather than its balanced bipartition, so the bipartite binding number is well defined.

Proposition 2.1. Let $G$ be a balanced bipartite graph. Then the value of $B(G)$ is independent of the choice of balanced bipartition.

Proof. If $G$ is connected, then the choice of balanced bipartition is unique (up to permutation of $V_{1}$ and $V_{2}$ ), so the statement holds trivially. It remains to consider the case when $G$ is disconnected.

Let $\left(V_{1}, V_{2}\right)$ be a balanced bipartition of $G$ such that the value of $B(G)$ is minimized (let $c$ denote this minimum value) and, subject to this, a corresponding binding set $S$ has smallest possible size. We claim that $S$ is entirely contained in one component of $G$; for otherwise, let $G_{1}, G_{2}, \ldots, G_{k}$ be all components of $G$ that intersect $S$, where $k \geq 2$, and set $S_{i}:=S \cap V\left(G_{i}\right)$ for $1 \leq i \leq k$. From the minimality assumption on $|S|$, we deduce that $\left|N\left(S_{i}\right)\right|>c\left|S_{i}\right|$ for all $i$ and hence $c|S|=\sum_{i=1}^{k} c\left|S_{i}\right|<\sum_{i=1}^{k}\left|N\left(S_{i}\right)\right|=|N(S)|=c|S|$; this contradiction justifies the claim. It follows that for any balanced bipartition $\left(U_{1}, U_{2}\right)$ of $G$, either $S \subseteq U_{1}$ or $S \subseteq U_{2}$. Therefore, $S$ is also a binding set of $G$ with respect to bipartition $\left(U_{1}, U_{2}\right)$.

Proposition 2.2. Every balanced bipartite graph $G$ with $B(G)>1$ is connected.

Let us now illustrate bipartite binding numbers using two special classes of graphs.

Proposition 2.3. $B\left(C_{2 n}\right)=\frac{n-1}{n-2}$ for $n \geq 3$.

Proof. Let $\left(V_{1}, V_{2}\right)$ be the bipartition of $C_{2 n}$, and let $S$ be a nonempty subset of $V_{i}, i=1$ or 2 , with $|N(S)|<n$. From the structure of $C_{2 n}$, we see that $|S| \leq n-2$ and $|S|<|N(S)|$. Hence

$$
\frac{|N(S)|}{|S|} \geq \frac{|S|+1}{|S|}=1+\frac{1}{|S|} \geq 1+\frac{1}{n-2}=\frac{n-1}{n-2}
$$

with equality when $S=V_{i}-\{u, v\}$, where $u$ and $v$ are two vertices in $V_{i}$ of distance 2 on $C_{2 n}$. So the statement is established.

Let $s$ and $t$ be two positive integers, and let $s K_{2} \oplus t \bar{K}_{2}$ be the bipartite graph obtained from the union of $s$ disjoint edges $a_{i} b_{i}$ for $1 \leq i \leq s$ by adding $2 t$ vertices $c_{1}, c_{2}, \ldots, c_{t}, d_{1}, d_{2}, \ldots, d_{t}$ and adding edges $a_{i} d_{j}$ and $b_{i} c_{j}$ for all $1 \leq i \leq s$ and $1 \leq j \leq$ $t$ (see Figure 2.1). For convenience, set $A:=\left\{a_{1}, a_{2}, \ldots, a_{s}\right\}, B:=\left\{b_{1}, b_{2}, \ldots, b_{s}\right\}$, $C:=\left\{c_{1}, c_{2}, \ldots, c_{t}\right\}$, and $D:=\left\{d_{1}, d_{2}, \ldots, d_{t}\right\}$. Clearly, $s K_{2} \oplus t \bar{K}_{2}$ has a unique bipartition $\left(V_{1}, V_{2}\right)$, where $V_{1}=A \cup C$ and $V_{2}=B \cup D$.

Proposition 2.4. Let $s$ and $t$ be two positive integers. Then

$$
B\left(s K_{2} \oplus t \bar{K}_{2}\right)= \begin{cases}\frac{1}{t} & \text { if } s=1, \\ \min \left\{\frac{s}{t}, \frac{s-1+t}{s-1}\right\} & \text { if } s>1 .\end{cases}
$$

Proof. Let $G=s K_{2} \oplus t \bar{K}_{2}$ and let $S$ be a binding set of $G$. Symmetry allows us to assume that $S \subseteq V_{1}$. Thus $|N(S)|<\left|V_{2}\right|$ by definition.

If $s=1$, then $a_{1} \notin S$. So $S \subseteq C$ and $N(S)=\left\{b_{1}\right\}$. As $S$ is a binding set of $G$, we must have $S=C$. Therefore, $B(G)=|N(S)| /|S|=1 / t$. 


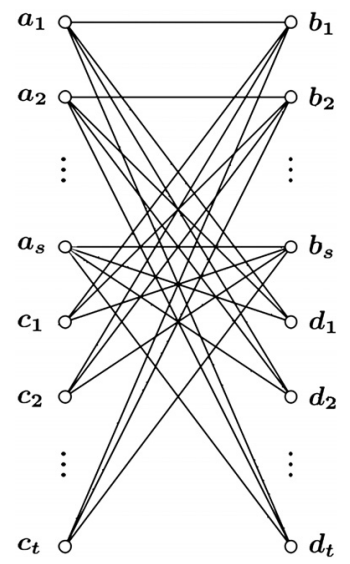

FIG. 2.1. $s K_{2} \oplus t \bar{K}_{2}$.

If $s>1$, then $A-S \neq \emptyset$. Furthermore, $S \cap A=\emptyset$ provided $S \cap C \neq \emptyset$, for otherwise we would have $N(S)=V_{2}$, a contradiction. It follows that $S$ is either a proper subset of $A$ or a subset of $C$. Thus $|N(S)|$ equals $|S|+t$ in the former case and $s$ in the latter case. As $S$ is a binding set of $G$, either $S=A-\left\{a_{i}\right\}$ for some $1 \leq i \leq s$ or $S=C$. From the definition we further deduce that $B(G)=|N(S)| /|S|=\min \left\{\frac{s}{t}, \frac{s-1+t}{s-1}\right\}$, completing the proof.

The following proposition asserts that the bound $3 / 2$ in Theorem 1.3 is indeed the threshold for a balanced bipartite graph to be Hamiltonian or bipancyclic.

Proposition 2.5. Let $G=s K_{2} \oplus t \bar{K}_{2}$. Then $B(G)=3 / 2$ if $s=2 t+1$, and $G$ is not Hamiltonian if $s \geq 2 t+1$.

Proof. The first statement follows instantly from Proposition 2.4. If $s \geq 2 t+1$, then $G-(C \cup D)$ contains precisely $s$ components (see Figure 2.1) with $s>|C \cup D|$. It follows that $G$ contains no Hamiltonian cycle. number.

The following lemma gives an alternative definition of the bipartite binding

Lemma 2.6. Let $G=\left(V_{1}, V_{2}, E\right)$ be a balanced bipartite graph with $2 n$ vertices. If $G \neq K_{n, n}$, then $B(G)$ is the largest nonnegative number $c$ such that

$$
c|N(S)| \geq(c-1) n+|S|
$$

for every nonempty subset $S$ of $V_{i}(i=1,2)$.

Proof. By definition, it suffices to show that for any given constant $c \geq 0$, the following two statements are equivalent:

(a) $c|N(S)| \geq(c-1) n+|S|$ for every nonempty $S \subseteq V_{i}$ and $i=1,2$;

(b) $|N(S)| \geq \min \{c|S|, n\}$ for every nonempty $S \subseteq V_{i}$ and $i=1,2$.

To this end, let $S$ be a nonempty subset of $V_{i}$ for $i=1$ or 2 , and let $T:=$ $V_{3-i}-N(S)$. Then $N(T)$ and $S$ are disjoint subsets of $V_{i}$, so $|N(T)|+|S| \leq n$ and hence

(c) $|N(T)| \leq n-|S| \leq n-1$.

If (a) holds, then (with $T$ in place of $S$ ) either $c|N(T)| \geq(c-1) n+|T|=c n-$ $|N(S)|$ or $T=\emptyset$. In the former case, $|N(S)| \geq \min \{c(n-|N(T)|), n\} \geq \min \{c|S|, n\}$ by (c). In the latter case, $V_{3-i}-N(S)=\emptyset$. So $|N(S)|=n \geq \min \{c|S|, n\}$. Combining these two cases, we obtain (b). 
Conversely, if (b) holds, then (with $T$ in place of $S$ ) either $|N(T)| \geq \min \{c|T|, n\}=$ $\min \{c n-c|N(S)|, n\}$ or $T=\emptyset$. In the former case, $c|N(S)| \geq c n-|N(T)| \geq$ $(c-1) n+|S|$ by (c). In the latter case, $V_{3-i}-N(S)=\emptyset$. So $|N(S)|=n$ and hence $c|N(S)|=c n \geq(c-1) n+|S|$. Combining these two cases, we establish (a).

As usual, we use $\delta(G)$ to denote the minimum degree of a graph $G$. The above lemma yields a lower bound on $\delta(G)$ when restricted to $|S|=1$.

Corollary 2.7. Let $G=\left(V_{1}, V_{2}, E\right)$ be a balanced bipartite graph with $2 n$ vertices. If $B(G) \geq c>0$, then

$$
\delta(G) \geq \frac{(c-1) n+1}{c} .
$$

Lemma 2.8. Let $G=\left(V_{1}, V_{2}, E\right)$ be a balanced bipartite graph with $2 n$ vertices. If $B(G)>n / 2$, then

$$
|N(S)| \geq\lceil(n+2|S|+1) / 3\rceil
$$

for every nonempty proper subset $S$ of $V_{i}(i=1,2)$.

Proof. As the statement holds trivially if $G=K_{n, n}$, we assume hereafter that $G \neq K_{n, n}$. Let $B(G)=c$ and let $S$ be a nonempty proper subset of $V_{i}$ for $i=1,2$. By Lemma 2.6, we have

$$
|N(S)| \geq \frac{(c-1) n+|S|}{c}=n-\frac{n-|S|}{c} .
$$

This together with $n-|S|>0$ and $c>3 / 2$ implies

$$
|N(S)|>n-\frac{2(n-|S|)}{3},
$$

and hence the desired statement holds.

The following lemma will play an important role in the subsequent proofs.

LEMMA 2.9. Let $G=\left(V_{1}, V_{2}, E\right)$ be a balanced bipartite graph with $2 n$ vertices and with $B(G)>3 / 2$. Let $X \subseteq V_{i}$ and $Y \subseteq V_{3-i}$, with $i=1$ or 2 , be nonempty sets such that $|X|,|Y|,|N(X)|$, and $|N(Y)|$ are all less than $n$. If $|Y| \geq|N(X)|-t$ for some nonnegative integer $t$, then $|N(Y)| \geq|X|+(2 n+4) / 5-t$.

Proof. Symmetry allows us to assume that $i=1$. For $S=X, Y$, by Lemma 2.8 and the definition of $B(G)$, we have

$$
|N(S)| \geq \max \left\{\frac{n+2|S|+1}{3}, \frac{3|S|+1}{2}\right\} .
$$

It follows that

$$
|Y| \geq|N(X)|-t \geq \max \left\{\frac{n+2|X|+1}{3}-t, \frac{3|X|+1}{2}-t\right\} .
$$

Plugging this inequality into (2.1) (with $S=Y$ ), we obtain

$$
|N(Y)| \geq \max \left\{\frac{n+2\left(\frac{3|X|+1}{2}-t\right)+1}{3}, \frac{3\left(\frac{n+2|X|+1}{3}-t\right)+1}{2}\right\} .
$$

Consequently,

$$
|N(Y)| \geq|X|+\max \{f(t), g(t)\}-t,
$$

Copyright $@$ by SIAM. Unauthorized reproduction of this article is prohibited. 


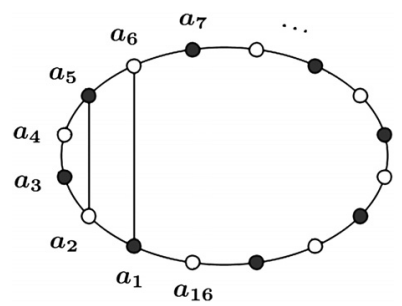

FIG. 3.1. $C_{16}^{2}$.

where $f(x):=(n+x+2) / 3$ and $g(x):=(n-x+2) / 2$. Observe that $f(x)$ is an increasing function of $x$, while $g(x)$ is a decreasing function of $x$, and that $f\left(x_{0}\right)=$ $g\left(x_{0}\right)=(2 n+4) / 5$, with $x_{0}=(n+2) / 5$. Hence $\max \{f(x), g(x)\} \geq f\left(x_{0}\right)$ for all $x$. Therefore $|N(Y)| \geq|X|+f\left(x_{0}\right)-t=|X|+(2 n+4) / 5-t$, as desired.

3. Nested cycle structures. Let $k$ and $m$ be two positive integers with $k \geq$ $m+2$, let $C=a_{1} a_{2} \ldots a_{2 k} a_{1}$ be a cycle of length $k$, where $a_{i+1}=a_{i}^{+}$for each $i$ (with $a_{2 k+1}=a_{1}$ ), and let $D$ be obtained from $C$ by adding $m$ chords $a_{i} a_{2 m+3-i}$ for $1 \leq i \leq m$. We write $D$ as $\overline{a_{1} a_{2} \ldots a_{2 m+2}} a_{2 m+3} \ldots a_{2 k} a_{1}$ and denote any graph isomorphic to $D$ by $C_{2 k}^{m}$. (See Figure 3.1 for $C_{16}^{2}$.) Observe that $C_{2 k}^{m}$ contains $m+1$ nested cycles $C_{2 k}, C_{2 k-2}, \ldots, C_{2 k-2 m}$ simultaneously. Intuitively, $C_{2 k}^{m}$ can be viewed as a ladder with $m$ rungs; our proof will rely heavily on such ladders. For any vertex $v$ on $D$, define $v^{+}:=v_{C}^{+}$and $v^{-}:=v_{C}^{-}$. For any two vertices $u$ and $v$ on $D$, define $u \vec{D} v:=u \vec{C} v$ and $D[u, v]:=C[u, v]$, etc.

To establish the main result, we first show the existence of $C_{4}, C_{6}^{1}$, and one of $C_{8}^{2}$, $C_{10}^{2}$, and $C_{12}^{2}$. The following statement and its proof are inspired by its counterparts on general graphs due to Reiman [5].

LEMma 3.1. Let $G=\left(V_{1}, V_{2}, E\right)$ be a balanced bipartite graph with $2 n$ vertices. If $|E|>n / 2(1+\sqrt{4 n-3})$, then $G$ contains a $C_{4}$.

Proof. Suppose $G$ contains no $C_{4}$. Consider triples of the form $(x,\{y, z\})$ such that $x \in V_{1}, y, z \in V_{2}$ with $y \neq z$, and that $x$ is adjacent to both $y$ and $z$. Since $G$ contains no $C_{4}$, each pair $\{y, z\}$ gives rise to at most one such triple. Hence the number of such triples is at most $\left(\begin{array}{l}n \\ 2\end{array}\right)$.

On the other hand, since each $x \in V_{1}$ gives rise to exactly $\left(\begin{array}{c}d(x) \\ 2\end{array}\right)$ such triples, the number of triples of the above form is equal to $\sum_{x \in V_{1}}\left(\begin{array}{c}d(x) \\ 2\end{array}\right)$. Let $\sigma=\sum_{x \in V_{1}} d(x) / n$. Then $\sigma=|E| / n$. As the extended binomial coefficient $\left(\begin{array}{l}t \\ 2\end{array}\right)$ is a convex function, by definition $\left(\begin{array}{c}\sigma \\ 2\end{array}\right) \leq \frac{1}{n} \sum_{x \in V_{1}}\left(\begin{array}{c}d(x) \\ 2\end{array}\right)$. So $\left(\begin{array}{c}\sigma \\ 2\end{array}\right) \leq \frac{1}{n}\left(\begin{array}{c}n \\ 2\end{array}\right)$ and hence $\sigma^{2}-\sigma-(n-1) \leq 0$. Solving this inequality yields $\sigma \leq 1 / 2(1+\sqrt{4 n-3})$. Therefore $|E| \leq n / 2(1+\sqrt{4 n-3})$, a contradiction.

Lemma 3.2. Let $G=\left(V_{1}, V_{2}, E\right)$ be a balanced bipartite graph with $2 n$ vertices. If $B(G)>3 / 2$ and $n>3$, then $G$ contains a $C_{4}$. implies

Proof. By Lemma 2.8, we have $\delta(G) \geq\lceil(n+3) / 3\rceil$. This together with $n>3$

$$
|E| \geq n \delta \geq n\lceil(n+3) / 3\rceil>\frac{n}{2}(1+\sqrt{4 n-3}) .
$$

Thus the statement follows instantly from Lemma 3.1. 
By Propositions 2.3 and 2.4, $C_{6}$ and $3 K_{2} \oplus \bar{K}_{2}$ have bipartite binding numbers 2 and $3 / 2$, respectively, yet neither of them contains a $C_{4}$. So the figures in the above lemma are both sharp.

Lemma 3.3. Let $G=\left(V_{1}, V_{2}, E\right)$ be a balanced bipartite graph with $2 n$ vertices. If $B(G)>3 / 2$ and $n \geq 10$, then $G$ contains a $C_{6}^{1}$.

Proof. By Lemma 3.2, $G$ contains a cycle $x_{1} y_{1} x_{2} y_{2} x_{1}$ of length 4 with $X=$ $\left\{x_{1}, x_{2}\right\} \subseteq V_{1}$ and $Y=\left\{y_{1}, y_{2}\right\} \subseteq V_{2}$. For $i=1,2$, define $X_{i}=N\left(y_{i}\right)-X$ and $Y_{i}=N\left(x_{i}\right)-Y$.

Assume on the contrary that $G$ contains no $C_{6}^{1}$. Then there is no edge between $X_{1} \cup X_{2}$ and $Y_{1} \cup Y_{2}$. Furthermore, $x_{1} \notin N\left(Y_{2}\right)$ or $y_{1} \notin N\left(X_{2}\right)$. Symmetry allows us to assume that $x_{1} \notin N\left(Y_{2}\right)$. Thus $Y_{1} \cap Y_{2}=\emptyset$. By Lemma 2.8, we obtain $\left|X_{1}\right| \geq\lceil(n+3) / 3\rceil-2=\lceil(n-3) / 3\rceil$, and the same is true for $\left|Y_{1}\right|$ and $\left|Y_{2}\right|$. Hence $\left|Y_{1} \cup Y_{2}\right| \geq 2\lceil(n-3) / 3\rceil$.

As $X_{1}$ is nonempty and $X_{1} \cap N\left(Y_{1} \cup Y_{2}\right)=\emptyset$, we have

$$
\begin{aligned}
n & \geq\left|X_{1}\right|+\left|N\left(Y_{1} \cup Y_{2}\right)\right| \\
& >\lceil(n-3) / 3\rceil+\frac{3}{2} \cdot 2\lceil(n-3) / 3\rceil,
\end{aligned}
$$

so $n \geq 4\lceil(n-3) / 3\rceil+1$ and hence $n \geq 4(n-3) / 3+1$, which implies $n \leq 9$, a contradiction.

LEMma 3.4. Let $G=\left(V_{1}, V_{2}, E\right)$ be a balanced bipartite graph with $2 n$ vertices. If $B(G)>3 / 2$ and $n \geq 14$, then $G$ contains at least one of $C_{8}^{2}, C_{10}^{2}$, and $C_{12}^{2}$.

Proof. Let $D=\overline{x_{1} y_{1} x_{2} y_{2}} x_{3} y_{3} x_{1}$ be a $C_{6}^{1}$ in $G$; the existence of $D$ is guaranteed by Lemma 3.3. Recall the definition that $x_{1} y_{2}$ is an edge in $D$. Set $X:=\left\{x_{1}, x_{2}, x_{3}\right\}$ and $Y:=\left\{y_{1}, y_{2}, y_{3}\right\}$. Symmetry allows us to assume that $X \subseteq V_{1}$ and $Y \subseteq V_{2}$. Define $N_{1}\left(x_{3}\right)=N\left(x_{3}\right)-Y, N_{2}\left(x_{3}\right)=N\left(N_{1}\left(x_{3}\right)\right)-X$, and $N_{3}\left(x_{3}\right)=N\left(N_{2}\left(x_{3}\right)\right)-Y$. Define $N_{i}\left(y_{3}\right)$ symmetrically for $1 \leq i \leq 3$.

Assume on the contrary that $G$ contains none of $C_{8}^{2}, C_{10}^{2}$, and $C_{12}^{2}$. We propose to show that

$$
N\left(N_{i}\left(x_{3}\right)\right) \cap N_{j}\left(y_{3}\right)=\emptyset \text { for all } 1 \leq i, j \leq 3 .
$$

Otherwise, let $(i, j)$ be a pair such that $N\left(N_{i}\left(x_{3}\right)\right) \cap N_{j}\left(y_{3}\right) \neq \emptyset$ and, subject to this, $i+j$ is minimum. Then $i \equiv j(\bmod 2)$ and $G\left[\left\{x_{3}, y_{3}\right\} \cup\left(\cup_{s=1}^{i} N_{s}\left(x_{3}\right)\right) \cup\left(\cup_{t=1}^{j} N_{t}\left(y_{3}\right)\right)\right]$ contains an $\left(x_{3}, y_{3}\right)$-path $\pi$ of length $i+j+1$. It follows that $\frac{y_{3} x_{1} y_{1} x_{2} y_{2} x_{3}}{C_{3}} y_{3}$ is a $C_{6+i+j}^{2}$ in $G$, and this contradiction establishes (3.1).

By taking $i=j=1$ in (3.1), we see that $N_{2}\left(x_{3}\right) \cap N_{1}\left(y_{3}\right)=\emptyset$, so $y_{3} \notin N\left(N_{2}\left(x_{3}\right)\right)$. Repeated application of Lemma 2.8 yields

$$
\begin{aligned}
\left|N_{1}\left(x_{3}\right)\right| & \geq\lceil(n+3) / 3\rceil-|Y| \geq(n-6) / 3, \\
\left|N_{2}\left(x_{3}\right)\right| & \geq\left\lceil\left(n+2\left|N_{1}\left(x_{3}\right)\right|+1\right) / 3\right\rceil-|X| \geq(5 n-36) / 9, \\
\left|N_{3}\left(x_{3}\right)\right| & \geq\left\lceil\left(n+2\left|N_{2}\left(x_{3}\right)\right|+1\right) / 3\right\rceil-\left|Y-\left\{y_{3}\right\}\right| \geq(19 n-117) / 27, \text { and } \\
\left|N\left(N_{3}\left(x_{3}\right)\right)\right| & \geq\left\lceil\left(n+2\left|N_{3}\left(x_{3}\right)\right|+1\right) / 3\right\rceil \geq(65 n-207) / 81 .
\end{aligned}
$$

Similarly, $\left|N_{3}\left(y_{3}\right)\right| \geq(19 n-117) / 27$. In view of (3.1), $N\left(N_{3}\left(x_{3}\right)\right)$ and $N_{3}\left(y_{3}\right)$ are disjoint subsets of $V_{1}$, so $\left|N\left(N_{3}\left(x_{3}\right)\right)\right|+\left|N_{3}\left(y_{3}\right)\right| \leq n$, which implies $41 n \leq 558$ and hence $n<14$, contradicting the hypothesis.

Let us digress briefly to introduce a term and make some simple observations, which will be used to show the existence of the aforementioned ladders. 
Let $D=\overline{a_{1} a_{2} \ldots a_{6}} a_{7} \ldots a_{2 k} a_{1}$ be a $C_{2 k}^{2}$, where $k \geq 4$ and $a_{1} \in V_{1}(D)$. A family $\left(A_{0}, A_{1}, \ldots, A_{t}\right)$, with $2 \leq t \leq 4$, of subsets of $V(D)$ is called good if the following two conditions are satisfied:

- $A_{0} \cup A_{1}^{+} \cup \cdots \cup A_{t}^{+t} \subseteq V_{2}(D)$ and

- $A_{i}^{+i} \cap A_{j}^{+j} \subseteq\left\{v \in V_{2}(D):\left\{v^{-i}, v^{-j}\right\} \cap D\left[a_{2}, a_{5}\right] \neq \emptyset\right\}$ for all $0 \leq i<j \leq t$.

Lemma 3.5. Suppose $\left(A_{0}, A_{1}, \ldots, A_{t}\right)$ is good. Then the following statements hold:

(i) If $t=4$ and $a_{6} \notin A_{2}^{+2} \cap A_{3}^{+3}$, then $\left|A_{0}\right|+\sum_{s=2}^{4}\left|A_{s}\right| \leq k+7$.

(ii) If $t \in\{2,3\}$, then $\sum_{s=0}^{t}\left|A_{s}\right| \leq k+\lceil 5 t / 2\rceil$.

Proof. Since $\left(A_{0}, A_{1}, \ldots, A_{t}\right)$ is a good family of subsets of $V(D)$, it is a routine matter to check using the definition that (where $A_{i}^{+i}$ exists only when $t \geq i$ for each $i$ )

(1) $A_{0} \cap A_{1}^{+}$and $A_{0} \cap A_{2}^{+2}$ are both subsets of $\left\{a_{2}, a_{4}, a_{6}\right\}$;

(2) $A_{0} \cap A_{3}^{+3}$ and $A_{0} \cap A_{4}^{+4}$ are both subsets of $\left\{a_{2}, a_{4}, a_{6}, a_{8}\right\}$;

(3) $A_{1}^{+} \cap A_{2}^{+2}$ is a subset of $\left\{a_{4}, a_{6}\right\}$;

(4) $A_{1}^{+} \cap A_{3}^{+3}, A_{2}^{+2} \cap A_{3}^{+3}, A_{1}^{+} \cap A_{4}^{+4}$, and $A_{2}^{+2} \cap A_{4}^{+4}$ are all subsets of $\left\{a_{4}, a_{6}, a_{8}\right\}$; and

(5) $A_{3}^{+3} \cap A_{4}^{+4}$ is a subset of $\left\{a_{6}, a_{8}\right\}$.

In the remainder of our proof, we use $f(v)$ to denote the number of sets in $\left\{A_{0}, A_{2}^{+2}\right.$, $\left.A_{3}^{+3}, A_{4}^{+4}\right\}$ if $t=4$ and in $\left\{A_{0}, A_{1}^{+}, \ldots, A_{t}^{+t}\right\}$ if $t \in\{2,3\}$ that contain a vertex $v$.

(i) By (4) and (5), $a_{2}$ is contained in at most one set in $\left\{A_{2}^{+2}, A_{3}^{+3}, A_{4}^{+4}\right\}$, so $f\left(a_{2}\right) \leq 2$. By $(5)$, we have $f\left(a_{4}\right) \leq 3$. By hypothesis, $a_{6} \notin A_{2}^{+2} \cap A_{3}^{+3}$. So $f\left(a_{6}\right) \leq 3$. From (1) we deduce that $f\left(a_{8}\right) \leq 3$. For all vertices $v \in$ $V_{2}(D)-\left\{a_{2}, a_{4}, a_{6}, a_{8}\right\}$, from (1), (2), (4), and (5) we see that $f(v) \leq 1$. Combining the above observations, we obtain

$$
\left|A_{0}\right|+\left|A_{2}^{+2}\right|+\left|A_{3}^{+3}\right|+\left|A_{4}^{+4}\right|=\sum_{v \in V_{2}(D)} f(v) \leq\left|V_{2}(D)\right|+7=k+7 .
$$

Thus (i) is established.

(ii) Let us consider the case when $t=2$. By (3), we have $a_{2} \notin A_{1}^{+} \cap A_{2}^{+2}$. So $f\left(a_{2}\right) \leq 2$. Clearly, $f\left(a_{4}\right) \leq 3$ and $f\left(a_{6}\right) \leq 3$. Moreover, from (1) and (3) we deduce that $f(v) \leq 1$ for all $v \in V_{2}(D)-\left\{a_{2}, a_{4}, a_{6}\right\}$. Hence

$$
\left|A_{0}\right|+\left|A_{1}^{+}\right|+\left|A_{2}^{+2}\right|=\sum_{v \in V_{2}(D)} f(v) \leq\left|V_{2}(D)\right|+5=k+\lceil 5 t / 2\rceil .
$$

It remains to consider the case when $t=3$. By (3) and (4), $a_{2}$ is contained in at most one set in $\left\{A_{1}^{+}, A_{2}^{+2}, A_{3}^{+3}\right\}$. So $f\left(a_{2}\right) \leq 2$. Clearly, $f\left(a_{4}\right) \leq 4$ and $f\left(a_{6}\right) \leq 4$. From (1) and (3), we see that $a_{8}$ is contained in at most one set in $\left\{A_{0}, A_{1}^{+}, A_{2}^{+2}\right\}$, so $f\left(a_{8}\right) \leq 2$. Moreover, for all vertices $v \in V_{2}(D)-\left\{a_{2}, a_{4}, a_{6}, a_{8}\right\}$, from (1)-(4) we deduce that $f(v) \leq 1$. Therefore,

$$
\sum_{s=0}^{3}\left|A_{s}\right|=\sum_{v \in V_{2}(D)} f(v) \leq\left|V_{2}(D)\right|+8=k+\lceil 5 t / 2\rceil .
$$

This completes the proof of the present lemma.

LEMMA 3.6. Let $G=\left(V_{1}, V_{2}, E\right)$ be a balanced bipartite graph with $2 n$ vertices, let $D=\overline{a_{1} a_{2} \ldots a_{6}} a_{7} \ldots a_{2 k} a_{1}$ be a $C_{2 k}^{2}$ in $G$ with $k \geq 4$, and let $X_{0}, X_{1}, \ldots, X_{t}$ be disjoint subsets of $V(G-D)$ with $t \in\{3,4\}$ such that 
(i) $X_{0}=\left\{x_{0}\right\}$, where $\left\{x_{0}, a_{1}\right\} \subseteq V_{1}$;

(ii) $\left|X_{1}\right|=1$ if $t=4$; and

(iii) $X_{i} \subseteq N\left(X_{i-1}\right)$ for $1 \leq i \leq t$.

Suppose $u$ is a vertex in $N_{D}\left(x_{0}\right)-\left\{a_{2}, a_{4}\right\}$. Let $A_{0}:=N_{D}\left(u^{+}\right)-\left\{u^{+2}, u^{+2(t-2)}\right\}$ and $A_{i}:=N_{D}\left(X_{i}\right)$ for $1 \leq i \leq t$. If $G$ contains none of $C_{2 k+2}^{2}, C_{2 k+4}^{2}$, and $C_{2 k+6}^{2}$, then $\left(A_{0}, A_{1}, \ldots, A_{t}\right)$ is a good family of subsets of $V(D)$.

Proof. Assume the contrary: there exist $0 \leq i<j \leq t$ and $v \in A_{i}^{+i} \cap A_{j}^{+j}$ such that $\left\{v^{-i}, v^{-j}\right\} \cap D\left[a_{2}, a_{5}\right]=\emptyset$. Set $H:=G-D$.

Let us first consider the case when $i=0$. Now $v \in N_{D}\left(u^{+}\right)-\left\{u^{+2}, u^{+2(t-2)}\right\}$, $v^{-j} \in N_{D}\left(X_{j}\right)$, and $\left\{v, v^{-j}\right\} \cap D\left[a_{2}, a_{5}\right]=\emptyset$. Observe that both $u$ and $v$ are in $V_{2}$ and $u \notin D\left(v^{-j}, v\right)$ (for otherwise $v=u^{+2}$ and $j \in\{3,4\}$, a contradiction). Let $x_{j}$ be a neighbor of $v^{-j}$ in $X_{j}$ and let $P$ be an $\left(x_{0}, x_{j}\right)$-path of length $j$ in $H\left[\cup_{s=0}^{j} X_{s}\right]$. Since $u \in V_{2}(D)$ and $v \in A_{0} \subseteq V_{2}(D)-\left\{u^{+2}, u^{+2(t-2)}\right\}$, we have $v^{-j} \neq u$ and hence $u \notin D\left[v^{-j}, v\right)$. This together with $\left\{v, v^{-j}\right\} \cap D\left[a_{2}, a_{5}\right]=\emptyset$ implies that either $D\left[v^{-j}, v\right] \subseteq D\left[u^{+}, a_{1}\right]$ or $D\left[v^{-j}, v\right] \subseteq D\left[a_{6}, u\right]$. Therefore

$$
D^{\prime}= \begin{cases}\overline{a_{1} a_{2} \ldots a_{6}} \vec{D} u x_{0} \vec{P} x_{j} v^{-j} \overleftarrow{D} u^{+} v \vec{D} a_{1} & \text { if } D\left[v^{-j}, v\right] \subseteq D\left[u^{+}, a_{1}\right] \\ \overline{a_{1} a_{2} \ldots a_{6}} \vec{D} v^{-j} x_{j} \overleftarrow{P} x_{0} u \overleftarrow{D} v u^{+} \vec{D} a_{1} & \text { if } D\left[v^{-j}, v\right] \subseteq D\left[a_{6}, u\right]\end{cases}
$$

is a $C_{2 k+2}^{2}$ in $G$, contradicting the hypothesis.

Next, let us consider the case when $i \geq 1$. Now $v^{-i} \in N_{D}\left(X_{i}\right)$ and $v^{-j} \in N_{D}\left(X_{j}\right)$. Let $x_{i}$ be a neighbor of $v^{-i}$ in $X_{i}$ and let $y_{j}$ be a neighbor of $v^{-j}$ in $X_{j}$. By (iii), $H\left[\cup_{s=t-3}^{i} X_{s}\right]$ contains a path $Q:=x_{t-3} x_{t-2} \ldots x_{i}$ of length $i-(t-3)$, where $x_{s} \in X_{s}$ for $t-3 \leq s \leq i$. Similarly, $H\left[\cup_{s=t-3}^{j} X_{s}\right]$ contains a path $R:=y_{t-3} y_{t-2} \ldots y_{j}$ of length $j-(t-3)$, where $y_{s} \in X_{s}$ for $t-3 \leq s \leq j$. Since $\left|X_{t-3}\right|=1$, we have $x_{t-3}=y_{t-3}$. Let $\ell$ be the largest subscript with $t-3 \leq \ell \leq i$ such that $x_{\ell}=y_{\ell}$. Then $0 \leq i-\ell \leq(t-1)-(t-3) \leq 2$. Set $S:=x_{i} \overleftarrow{Q} x_{\ell} \vec{R} y_{j}$. Clearly, $S$ is a path in $H\left[\cup_{s=t-3}^{j} X_{s}\right]$ of length $j-i+2(i-\ell)$. Thus we obtain a $C_{2 k+2(i-\ell)+2}^{2}$ from $D$ by replacing $v^{-j} \vec{D} v^{-i}$ with $v^{-j} y_{j} \overleftarrow{S} x_{i} v^{-i}$, contradicting the hypothesis again.

Our next two lemmas show that if $G$ contains a $C_{2 k}^{2}$, denoted by $D$, such that $G-D$ has a path with length at least three, then we can find a $C_{2 t}^{2}$ in $G$ based on the above two lemmas for some $t$ with $k+1 \leq t \leq k+3$.

Lemma 3.7. Let $G=\left(V_{1}, V_{2}, E\right)$ be a balanced bipartite graph with $2 n$ vertices, let $D=\overline{a_{1} a_{2} \ldots a_{6}} a_{7} \ldots a_{2 k} a_{1}$ be a $C_{2 k}^{2}$ in $G$, and let $x_{0} x_{1} x_{2} x_{3} x_{4}$ be a path in $G-D$ such that $N_{D}\left(x_{0}\right)-\left\{a_{2}, a_{3}, a_{4}, a_{5}\right\} \neq \emptyset$. If $B(G)>3 / 2, n \geq 139$, and $k \geq 4$, then $G$ contains at least one of $C_{2 k+2}^{2}, C_{2 k+4}^{2}$, and $C_{2 k+6}^{2}$.

Proof. Assume on the contrary that

$$
G \text { contains none of } C_{2 k+2}^{2}, C_{2 k+4}^{2} \text {, and } C_{2 k+6}^{2} \text {. }
$$

By Proposition 2.2, $G$ is connected. Symmetry allows us to assume that $x_{0}$ and $a_{1}$ are in the same color class of $G$, for otherwise rewrite $D$ as $\overline{b_{1} b_{2} \ldots b_{6}} b_{7} \ldots b_{2 k} b_{1}$, where $b_{i}=a_{7-i}$ for $1 \leq i \leq 6$. Then $x_{0}$ and $b_{1}$ are in the same class, as desired. Renaming subscripts of $V_{i}$ 's if necessary, we may assume that $\left\{x_{0}, a_{1}\right\} \subseteq V_{1}$.

Let $H=G-D$ and $u \in N_{D}\left(x_{0}\right)-\left\{a_{2}, a_{4}\right\}$. Define $X_{1}:=\left\{x_{1}\right\}, X_{2}:=\left\{x_{2}\right\}$, $X_{3}:=N_{H}\left(x_{2}\right)-\left\{x_{1}\right\}, X_{4}:=N_{H}\left(X_{3}\right)-\left\{x_{0}, x_{2}\right\}$, and $X_{5}:=N_{H}\left(X_{4}\right)-\left(X_{3} \cup\left\{x_{1}\right\}\right)$ (see Figure 3.2). Note that $X_{1}, X_{2}, X_{3}, X_{4}$ and $X_{5}$ are disjoint subsets of $V(H)-\left\{x_{0}\right\}$. By (3.2), we have $N_{H}\left(u^{+}\right) \cap\left(X_{1} \cup X_{3} \cup X_{5}\right)=\emptyset$, so $N_{H}\left(u^{+}\right), X_{1}, X_{3}$, and $X_{5}$ are disjoint subsets of $V_{2}(H)$, which implies that $\left|N_{H}\left(u^{+}\right)\right|+\left|X_{1}\right|+\left|X_{3}\right|+\left|X_{5}\right| \leq n-k$ and hence 


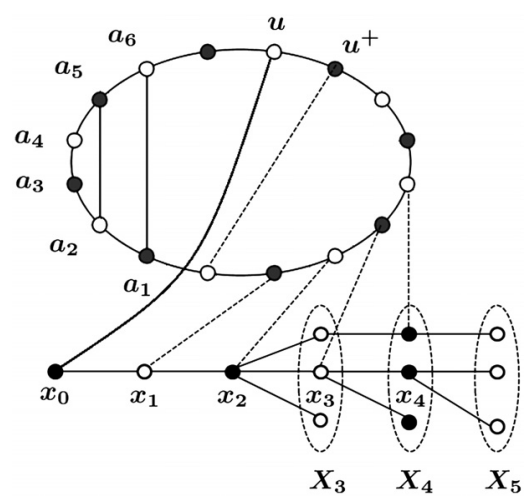

FIG. 3.2. $D$ and $X_{i}$ 's.

$$
\left|N_{H}\left(u^{+}\right)\right|+\left|N_{H}\left(x_{2}\right)\right|+\left|N_{H}\left(X_{4}\right)-\left(X_{3} \cup\left\{v_{1}\right\}\right)\right| \leq n-k .
$$

Set $A_{0}:=N_{D}\left(u^{+}\right)-\left\{u^{+2}, u^{+4}\right\}$ and $A_{i}:=N_{D}\left(X_{i}\right)$ for $1 \leq i \leq 4$. By Lemma 3.6,

$$
\left(A_{0}, A_{1}, A_{2}, A_{3}, A_{4}\right) \text { is a good family of subsets of } V(D) \text {. }
$$

Observe that

$$
a_{6} \notin A_{2}^{+2} \cap A_{3}^{+3},
$$

for otherwise $a_{4}$ is adjacent to $x_{2}$ and $a_{3}$ is adjacent to some vertex $x_{3}^{\prime}$ in $X_{3}$. It follows that $\overline{a_{2} a_{3} x_{3}^{\prime} x_{2} a_{4} a_{5}} a_{6} \vec{D} a_{2}$ is a $C_{2 k+2}^{2}$ in $G$, and this contradiction to (3.2) establishes (3.5).

From (3.4), (3.5), and Lemma 3.5, we deduce that $\left|A_{0}\right|+\left|A_{2}\right|+\left|A_{3}\right|+\left|A_{4}\right| \leq k+7$. Hence

$$
\left|N_{D}\left(u^{+}\right)\right|+\left|N_{D}\left(x_{2}\right)\right|+\left|N_{D}\left(X_{3}\right)\right|+\left|N_{D}\left(X_{4}\right)\right| \leq k+9 .
$$

Adding this inequality to (3.3) yields

$$
\left|N\left(u^{+}\right)\right|+\left|N\left(x_{2}\right)\right|+\left|N_{D}\left(X_{3}\right)\right|+\left|N\left(X_{4}\right)\right|-\left|X_{3}\right| \leq n+10 .
$$

By (3.2), we have $N_{D}\left(X_{3}\right) \subseteq V_{1}(D)-\left\{u^{+}\right\}$and $N_{D}\left(X_{4}\right) \subseteq V_{2}(D)-\left(\left\{u^{-2}, u^{+2}\right\}-\right.$ $\left.\left\{a_{2}, a_{4}\right\}\right)$, so $\left|N\left(X_{i}\right)\right|<n$ for $i=3$, 4 . As $\left|X_{4}\right|=\left|N_{H}\left(X_{3}\right)-\left\{x_{0}, x_{2}\right\}\right| \geq\left|N\left(X_{3}\right)\right|-$ $\left(\left|N_{D}\left(X_{3}\right)\right|+2\right)$, the triple $(X, Y, t)=\left(X_{3}, X_{4},\left|N_{D}\left(X_{3}\right)\right|+2\right)$ satisfies the hypothesis of Lemma 2.9 and hence

$$
\left|N\left(X_{4}\right)\right| \geq\left|X_{3}\right|+(2 n+4) / 5-\left(\left|N_{D}\left(X_{3}\right)\right|+2\right) .
$$

Combining this inequality with (3.6) gives $\left|N\left(u^{+}\right)\right|+\left|N\left(x_{2}\right)\right|+(2 n-6) / 5 \leq n+10$. Thus, by Lemma 2.8 we obtain $2(n+3) / 3+(2 n-6) / 5 \leq n+10$, which implies $n \leq 138$, and this contradiction completes the proof of our lemma.

Lemma 3.8. Let $G=\left(V_{1}, V_{2}, E\right)$ be a balanced bipartite graph with $2 n$ vertices, let $D=\overline{a_{1} a_{2} \ldots a_{6}} a_{7} \ldots a_{2 k} a_{1}$ be a $C_{2 k}^{2}$ in $G$, and let $x_{0} x_{1} x_{2} x_{3}$ be a path in $G-D$ such that $N_{D}\left(x_{0}\right)-\left\{a_{2}, a_{3}, a_{4}, a_{5}\right\} \neq \emptyset$. If $B(G)>3 / 2, n \geq 139$, and $k \geq 4$, then $G$ contains at least one of $C_{2 k+2}^{2}, C_{2 k+4}^{2}$, and $C_{2 k+6}^{2}$.

Copyright (c) by SIAM. Unauthorized reproduction of this article is prohibited. 
Proof. Assume on the contrary that

$$
G \text { contains none of } C_{2 k+2}^{2}, C_{2 k+4}^{2} \text {, and } C_{2 k+6}^{2} \text {. }
$$

By symmetry, we may assume that $\left\{x_{0}, a_{1}\right\} \subseteq V_{1}$. (See the first paragraph of the proof of the preceding lemma.)

Let $H=G-D$ and $u \in N_{D}\left(x_{0}\right)-\left\{a_{2}, a_{4}\right\}$. Define $X_{1}:=N_{H}\left(x_{0}\right)-\left\{x_{3}\right\}$, $X_{2}:=N_{H}\left(X_{1}\right)-\left\{x_{0}\right\}$, and $X_{3}:=N_{H}\left(X_{2}\right)-X_{1}$. If there exists a vertex $x_{4}$ in $N_{H}\left(x_{3}\right)-\left\{x_{0}, x_{2}\right\}$, then $x_{0} x_{1} x_{2} x_{3} x_{4}$ would be a path in $G-D$ and thus we reach a contradiction to (3.7) by Lemma 3.7. Therefore

$$
N_{H}\left(x_{3}\right) \subseteq\left\{x_{0}, x_{2}\right\} .
$$

Similarly,

$$
N_{H}\left(X_{3}\right) \subseteq\left\{x_{0}\right\} \cup X_{2} .
$$

By (3.7), we have $N_{H}\left(u^{+}\right) \cap\left(X_{1} \cup X_{3}\right)=\emptyset$, so $N_{H}\left(u^{+}\right), X_{1}$, and $X_{3}$ are disjoint subsets of $V_{2}(H)$. It follows that $\left|N_{H}\left(u^{+}\right)\right|+\left|X_{1}\right|+\left|X_{3}\right| \leq n-k$ and hence

$$
\left|N_{H}\left(u^{+}\right)\right|+\left|N_{H}\left(X_{2}\right)\right| \leq n-k .
$$

From Lemma 3.6, we see that $\left(N_{D}\left(u^{+}\right)-\left\{u^{+2}\right\}, N_{D}\left(X_{1}\right), N_{D}\left(X_{2}\right), N_{D}\left(X_{3}\right)\right)$ is a good family of subsets of $V(D)$. By Lemma 3.5, we thus obtain

$$
\left(\left|N_{D}\left(u^{+}\right)\right|-1\right)+\left|N_{D}\left(X_{1}\right)\right|+\left|N_{D}\left(X_{2}\right)\right|+\left|N_{D}\left(X_{3}\right)\right| \leq k+8 .
$$

Adding this inequality to (3.10) yields

$$
d\left(u^{+}\right)+\left|N_{D}\left(X_{1}\right)\right|+\left|N\left(X_{2}\right)\right|+\left|N_{D}\left(X_{3}\right)\right| \leq n+9 .
$$

In view of (3.7), we get $N_{D}\left(X_{1}\right) \subseteq V_{1}(D)-\left\{u^{+}\right\}$and $N_{D}\left(X_{2}\right) \subseteq V_{2}(D)-$ $\left(\left\{u^{-2}, u^{+2}\right\}-\left\{a_{2}, a_{4}\right\}\right)$. Hence $\left|N\left(X_{i}\right)\right|<n$ for $i=1,2$. As $\left|X_{2}\right|=\left|N_{H}\left(X_{1}\right)-\left\{x_{0}\right\}\right| \geq$ $\left|N\left(X_{1}\right)\right|-\left(\left|N_{D}\left(X_{1}\right)\right|+1\right)$, the triple $(X, Y, t)=\left(X_{1}, X_{2},\left|N_{D}\left(X_{1}\right)\right|+1\right)$ satisfies the hypothesis of Lemma 2.9 and hence

$$
\left|N\left(X_{2}\right)\right| \geq\left|X_{1}\right|+(2 n+4) / 5-\left(\left|N_{D}\left(X_{1}\right)\right|+1\right) .
$$

Combining this inequality with (3.11) gives

$$
d\left(u^{+}\right)+\left|X_{1}\right|+(2 n+4) / 5-1+\left|N_{D}\left(X_{3}\right)\right| \leq n+9 .
$$

Using (3.8), we obtain $\left|N_{D}\left(X_{3}\right)\right| \geq\left|N_{D}\left(x_{3}\right)\right|=\left|N\left(x_{3}\right)\right|-\left|N_{H}\left(x_{3}\right)\right| \geq d\left(x_{3}\right)-2$, so $d\left(u^{+}\right)+(2 n+4) / 5+\left(d\left(x_{3}\right)-2\right) \leq n+9$. From Lemma 2.8 , it follows that $(n+3) / 3+(2 n+4) / 5+(n+3) / 3-2 \leq n+9$. Therefore $n \leq 123$, and this contradiction completes the proof of our lemma.

4. A generalized bipartite hopping lemma. The hopping lemma was first introduced by Woodall [8] in his proof of Theorem 1.1, which demonstrates that the approach of iterating cycle exchanges can be highly effective for finding long cycles. Variations of the lemma were subsequently developed by various authors for use in different works. In particular, Ash [1] developed a basic version of the hopping lemma for bipartite graphs. 
The following lemma is an extract of results from Ash [1] (see Lemmas 4.3, 4.4, 4.9, and 4.16; see also Jackson and $\mathrm{Li}[4])$.

Lemma 4.1 (Ash [1]). Let $G=\left(V_{1}, V_{2}, E\right)$ be a bipartite graph, and let $C$ be a longest cycle of $G$ such that the number of components of $G-C$ is as small as possible and, subject to this, a smallest component $H$ of $G-C$ is as small as possible. Suppose there exist $a \in V_{1}-V(C)$ and $b \in V_{2}-V(C)$ such that either $a$ and $b$ are both isolated vertices in $G-C$ or $V(H)=\{a, b\}$. For each vertex $v$ in $G-C$, set $Y_{0}(v):=\emptyset$, and define recursively sets $X_{i}(v)$ and $Y_{i}(v)$ for $i \geq 1$ by $X_{i}(v):=N_{C}\left(Y_{i-1}(v) \cup\{v\}\right)$ and $Y_{i}(v):=\left\{y \in C: y^{-}, y^{+} \in X_{i}(v)\right\}$. Set $X_{v}:=\cup_{i \geq 1} X_{i}(v)$ and $Y_{v}:=\cup_{i \geq 1} Y_{i}(v)$. Then the following statements hold:

(i) $N\left(Y_{v}\right) \subseteq X_{v}$ for $v \in\{a, b\}$;

(ii) $X_{a} \cap Y_{b}=\emptyset=X_{b} \cap Y_{a}$;

(iii) $\left|X_{a}^{+} \cap X_{b}\right| \leq 1$ and $\left|X_{a}^{-} \cap X_{b}\right| \leq 1$; and

(iv) $X_{a}^{+} \cap X_{b}=\emptyset=X_{a}^{-} \cap X_{b}$ if $a b \in E$.

For convenience, set $C_{2 k}^{0}:=C_{2 k}$ for all $k \geq 2$. Observe that in Ash's lemma $C$ is assumed to be a longest cycle of $G$ under certain restrictions, while in our proof we need a generalized version which can be used to deal with the case when $G$ contains some $C_{2 k}^{m}$ (not necessarily a longest one) but no $C_{2 k+2}^{m}$ for $m \geq 0$ under some other restrictions. Let us now present this generalized bipartite hopping lemma, which ensures that the ladder structure can be preserved when growing a cycle.

Lemma 4.2. Let $G=\left(V_{1}, V_{2}, E\right)$ be a bipartite graph, and let $D=\overline{a_{1} a_{2} \ldots a_{2 m+2}}$ $a_{2 m+3} \ldots a_{2 k} a_{1}$ be a $C_{2 k}^{m}$ in $G$ with $m \geq 0$ and $a_{1} \in V_{1}$. Suppose $G$ contains neither $C_{2 k+2}^{m}$ nor another $C_{2 k}^{m}$, denoted by $D^{\prime}$, such that $G-D^{\prime}$ has fewer components than $G-D$, and suppose there exist $a \in V_{1}-V(D)$ and $b \in V_{2}-V(D)$ such that both of them are isolated vertices in $G-D$. For each vertex $v$ in $G-D$, set $Y_{0}(v):=\emptyset$, and define recursively sets $X_{i}(v)$ and $Y_{i}(v)$ for $i \geq 1$ by $X_{i}(v):=N_{D}\left(Y_{i-1}(v) \cup\{v\}\right)-$ $D\left(a_{1}, a_{2 m+2}\right)$ and $Y_{i}(v):=\left\{y \in D: y^{-}, y^{+} \in X_{i}(v)\right\}$, where $D\left(a_{1}, a_{2 m+2}\right)=\emptyset$ if $m=0$. Set $X_{v}:=\cup_{i \geq 1} X_{i}(v)$ and $Y_{v}:=\cup_{i \geq 1} Y_{i}(v)$. Then the following statements hold:

(i) $N\left(Y_{v}\right) \subseteq X_{v} \cup D\left(a_{1}, a_{2 m+2}\right)$ for $v \in\{a, b\}$;

(ii) $X_{a} \cap Y_{b}=\emptyset=X_{b} \cap Y_{a}$; and

(iii) $\left|X_{a}^{+} \cap X_{b}\right| \leq 1$ and $\left|X_{a}^{-} \cap X_{b}\right| \leq 1$.

Since the proof of this lemma is very tedious, we postpone it till section 6 so that the proof of our main theorem proceeds in a smoother and more coherent way. Clearly, the following monotonicity property holds for the objects defined in the above two lemmas:

$$
X_{1}(v) \subseteq X_{2}(v) \subseteq X_{3}(v) \subseteq \cdots \subseteq X_{v} \quad \text { and } \quad Y_{1}(v) \subseteq Y_{2}(v) \subseteq Y_{3}(v) \subseteq \cdots \subseteq Y_{v}
$$

As an application of the above generalized bipartite hopping lemma, let us derive the following statement, which will be used later.

LEMma 4.3. Let $G=\left(V_{1}, V_{2}, E\right)$ be a balanced bipartite graph with $2 n$ vertices, and let $D=\overline{a_{1} a_{2} \ldots a_{2 m+2}} a_{2 m+3} \ldots a_{2 k} a_{1}$ be $a C_{2 k}^{m}$ in $G$ with $m \geq 0$. Suppose $G$ does not contain another $C_{2 k}^{m}$, denoted by $D^{\prime}$, such that $G-D^{\prime}$ has fewer components than $G-D$, and suppose there exist $a \in V_{1}-V(D)$ and $b \in V_{2}-V(D)$ such that both of them are isolated vertices in $G-D$. If $B(G)>3 / 2$ and $m+2 \leq k \leq n-3 m-1$, then $G$ contains a $C_{2 k+2}^{m}$.

Proof. Assume the contrary: $G$ contains no $C_{2 k+2}^{m}$. Recall Lemma 4.2 and consider the sets $X_{i}(a)^{+}$and $X_{i}(a)^{-}$for $i \geq 1$. By (4.1) and Lemma 4.2(iii), each of $X_{i}(a)^{+}$and $X_{i}(a)^{-}$contains at most one vertex in $X_{i}(b)$. Hence $\left|X_{i}(a)^{+} \cap X_{i}(a)^{-}\right|=\left|X_{i}(a)^{+}\right|+$ 
$\left|X_{i}(a)^{-}\right|-\left|X_{i}(a)^{+} \cup X_{i}(a)^{-}\right| \geq\left|X_{i}(a)^{+}\right|+\left|X_{i}(a)^{-}\right|-\left|V_{1}(D)-X_{i}(b)\right|-2=\left(2\left|X_{i}(a)\right|-\right.$ $2)-\left(k-\left|X_{i}(b)\right|\right)$. By the definition of $Y_{i}(a)$, we have

$$
\left|Y_{i}(a)\right| \geq\left|X_{i}(a)^{+} \cap X_{i}(a)^{-}\right| \geq\left(2\left|X_{i}(a)\right|-2\right)-\left(k-\left|X_{i}(b)\right|\right) .
$$

Similarly,

$$
\left|Y_{i}(b)\right| \geq\left|X_{i}(b)^{+} \cap X_{i}(b)^{-}\right| \geq\left(2\left|X_{i}(b)\right|-2\right)-\left(k-\left|X_{i}(a)\right|\right) .
$$

Adding these two inequalities yields

$$
\left|Y_{i}(a)\right|+\left|Y_{i}(b)\right| \geq 3\left|X_{i}(a)\right|+3\left|X_{i}(b)\right|-2 k-4 .
$$

From the definition, (4.1), and Lemma 4.2(ii), it is clear that $Y_{i}(a) \subseteq V_{1}(D)-$ $D\left(a_{1}, a_{2 m+2}\right)-X_{1}(b)$. As $D\left(a_{1}, a_{2 m+2}\right) \cap V_{1}(D) \neq \emptyset$ if $m \geq 1$ and $X_{1}(b) \neq \emptyset$ if $m=0$, we have $Y_{i}(a) \neq V_{1}(D)$. Therefore $Y_{i}(a) \cup\{a\}$ is a proper subset of $V_{1}$ for all $i \geq 0$. Since $a$ is an isolated vertex of $G-D$, from the definition, (4.1), and Lemma 4.2(i), we deduce that $N\left(Y_{i-1}(a) \cup\{a\}\right) \subseteq X_{i}(a) \cup\left(V_{2} \cap D\left(a_{1}, a_{2 m+2}\right)\right)$. This together with Lemma 2.8 implies that

$$
\left|X_{i}(a)\right|+m \geq\left|N\left(Y_{i-1}(a) \cup\{a\}\right)\right| \geq \frac{n+2\left|Y_{i-1}(a)\right|+3}{3},
$$

so $3\left|X_{i}(a)\right| \geq n+2\left|Y_{i-1}(a)\right|-3(m-1)$. Similarly, $3\left|X_{i}(b)\right| \geq n+2\left|Y_{i-1}(b)\right|-3(m-1)$. Hence

$$
\begin{aligned}
\left|Y_{i}(a)\right|+\left|Y_{i}(b)\right| & \geq 2 n+2\left|Y_{i-1}(a)\right|+2\left|Y_{i-1}(b)\right|-6(m-1)-2 k-4 \\
& =2\left(\left|Y_{i-1}(a)\right|+\left|Y_{i-1}(b)\right|\right)+2(n-k)-6 m+2 \\
& \geq 2\left(\left|Y_{i-1}(a)\right|+\left|Y_{i-1}(b)\right|\right)+4 \quad(\text { as } k \leq n-3 m-1),
\end{aligned}
$$

which implies

$$
\left|Y_{i}(a)\right|+\left|Y_{i}(b)\right|+4 \geq 2\left(\left|Y_{i-1}(a)\right|+\left|Y_{i-1}(b)\right|+4\right) .
$$

Since $Y_{0}(a)=Y_{0}(b)=\emptyset$, it follows that $\left|Y_{i}(a)\right|+\left|Y_{i}(b)\right|+4 \geq 2^{i+2}$ for all $i \geq 1$, and hence $\left|Y_{i}(a)\right|+\left|Y_{i}(b)\right| \rightarrow \infty$ as $i \rightarrow \infty$, which is absurd.

5. Proof of Theorem 1.3. The proof of our theorem comes in three steps, and different steps require different counting techniques. Actually we have already carried out step 1 in section 3 by showing the existence of $C_{4}, C_{6}^{1}$ and one of $C_{8}^{2}, C_{10}^{2}$, and $C_{12}^{2}$ in $G$. Based on such a ladder and Lemma 4.3, we can now proceed to step 2, which aims to prove that $G$ contains a $C_{2 k}$ for every $k$ with $2 \leq k \leq n-6$.

Lemma 5.1. Let $G=\left(V_{1}, V_{2}, E\right)$ be a balanced bipartite graph with $2 n$ vertices and with a $C_{2 k}^{2}$. If $B(G)>3 / 2, n \geq 139$, and $4 \leq k \leq n-7$, then $G$ contains at least one of $C_{2 k+2}^{2}, C_{2 k+4}^{2}$, and $C_{2 k+6}^{2}$.

Proof. By hypothesis, $G$ contains a subgraph $D=\overline{a_{1} a_{2} \ldots a_{6}} a_{7} \ldots a_{2 k} a_{1}$ (with $a_{1} \in V_{1}$ ), which is a $C_{2 k}^{2}$. Assume on the contrary that $G$ contains none of $C_{2 k+2}^{2}$, $C_{2 k+4}^{2}$, and $C_{2 k+6}^{2}$. Let us make some simple observations about $G-D$.

Claim 1. No component $H$ of $G-D$ satisfies $\min \left\{\left|V_{1}(H)\right|,\left|V_{2}(H)\right|\right\} \geq 2$.

Suppose for a contradiction that $\min \left\{\left|V_{1}(H)\right|,\left|V_{2}(H)\right|\right\} \geq 2$ for some component $H$ of $G-D$. Then $H$ contains a path $x_{0} x_{1} x_{2} x_{3}$ with $x_{0} \in V_{1}(H)$. By Lemma 3.8, we have $N_{D}\left(x_{0}\right) \cup N_{D}\left(x_{3}\right) \subseteq D\left[a_{2}, a_{5}\right]$, so $N_{D}\left(x_{0}\right) \subseteq\left\{a_{2}, a_{4}\right\}$ and $N_{D}\left(x_{3}\right) \subseteq\left\{a_{3}, a_{5}\right\}$.

Copyright $\odot$ by SIAM. Unauthorized reproduction of this article is prohibited. 
Using Lemma 2.8, we obtain $\left|N_{H}\left(x_{i}\right)\right|=d\left(x_{i}\right)-\left|N_{D}\left(x_{i}\right)\right| \geq(n-3) / 3$ for $i=0,3$. Thus $\left|V_{i}(H)\right| \geq(n-3) / 3>45$ for $i=1,2$.

Symmetry allows us to assume that $\left|V_{1}(H)\right| \geq\left|V_{2}(H)\right|$. Let us show that there exist two distinct vertices $v_{1}$ and $v_{2}$ in $V_{1}(H)$ such that

$$
N_{D}\left(v_{i}\right)-\left\{a_{2}, a_{4}\right\} \neq \emptyset \text { for } i=1,2 .
$$

Otherwise, there is a subset $X$ of $V_{1}(H)$ with $|X| \geq\left|V_{1}(H)\right|-1$ such that $N_{D}(X) \subseteq$ $\left\{a_{2}, a_{4}\right\}$. So $|N(X)|<n$ and hence $\left|N_{H}(X)\right|=|N(X)|-\left|N_{D}(X)\right|>3|X| / 2-2 \geq$ $\left(3\left|V_{1}(H)\right|-7\right) / 2 \geq\left(3\left|V_{2}(H)\right|-7\right) / 2>\left|V_{2}(H)\right|$ as $V_{2}(H) \geq 45$, a contradiction. Therefore (5.1) is true.

Set $A:=N_{H}\left(v_{1}\right)$ and $B:=N_{H}(A)-\left\{v_{1}\right\}$. If $N_{H}(B)-A$ contains a vertex $z$, then letting $y \in N_{B}(z)$ and $x \in N_{A}(y)$, the path $v_{1} x y z$ is fully contained in $G-D$, contradicting Lemma 3.8. So $N_{H}(B) \subseteq A$, which in turn implies $V_{1}(H)=B \cup\left\{v_{1}\right\}$ and $V_{2}(H)=A$. Hence $|A| \geq(n-3) / 3>45$ and $v_{2} \in B$. Let $u_{2} \in N_{A}\left(v_{2}\right)$ and $u_{1} \in A-\left\{u_{2}\right\}$. Then $v_{2} u_{2} v_{1} u_{1}$ is a path in $G-D$, which contradicts Lemma 3.8. So Claim 1 is justified.

Claim 2. Each component of $G-D$ contains at most two vertices.

Suppose the contrary: some component $H$ of $G-D$ has at least three vertices. By Claim 1, we have $\min \left\{\left|V_{1}(H)\right|,\left|V_{2}(H)\right|\right\} \leq 1$. So $H$ is a star. By symmetry, we may assume that $V_{1}(H)=\left\{x_{1}, x_{2}, \ldots, x_{r}\right\}$ and $V_{2}(H)=\{y\}$, where $r \geq 2$. Set $X:=V_{1}(H)-\left\{x_{1}\right\}$. Since $G$ contains no $C_{2 k+2}^{2}$,

$$
\begin{aligned}
& N_{D}\left(x_{1}\right) \cap N_{D}^{+}(y) \subseteq\left\{a_{2}, a_{6}\right\}, \\
& N_{D}\left(x_{1}\right) \cap N_{D}^{+2}(X) \subseteq\left\{a_{2}, a_{4}, a_{6}\right\}, \text { and } \\
& N_{D}^{+}(y) \cap N_{D}^{+2}(X) \subseteq\left\{a_{4}, a_{6}\right\} .
\end{aligned}
$$

So each of $a_{2}$ and $a_{4}$ is contained in at most two sets in $\left\{N_{D}\left(x_{1}\right), N_{D}^{+2}(X), N_{D}^{+}(y)\right\}$. Consequently, $\left|N_{D}\left(x_{1}\right)\right|+\left|N_{D}^{+}(y)\right|+\left|N_{D}^{+2}(X)\right| \leq\left|V_{2}(D)-\left\{a_{2}, a_{4}, a_{6}\right\}\right|+7=k+4$. By Lemma 2.8, we have $\left|N_{D}\left(x_{1}\right)\right|=\left|N\left(x_{1}\right)-\{y\}\right| \geq n / 3,\left|N_{D}(y)\right|=|N(y)|-r \geq$ $(n+3) / 3-r$, and $\left|N_{D}(X)\right|=|N(X)-\{y\}| \geq(n+2|X|+1) / 3-1=(n+2 r-4) / 3$. Therefore, $n / 3+(n+3) / 3-r+(n+2 r-4) / 3 \leq k+4$, which implies that $3 n \leq$ $3 k+r+13 \leq n+2 k+13$ and hence $k>n-7$, contradicting the hypothesis. This proves Claim 2.

Claim 3. $G-D$ contains at most one isolated edge.

To justify this, we assume that both $x_{1} y_{1}$ and $x_{2} y_{2}$ are two isolated edges of $G-D$ with $\left\{x_{1}, x_{2}\right\} \subseteq V_{1}$ and $\left\{y_{1}, y_{2}\right\} \subseteq V_{2}$. We propose to show that

$$
\text { or } \quad \begin{aligned}
N_{D}\left(x_{1}\right) \cap N_{D}^{+2}\left(x_{2}\right) \subseteq\left\{a_{2}, a_{4}, a_{6}\right\} \\
N_{D}\left(y_{1}\right) \cap N_{D}^{+2}\left(y_{2}\right) \subseteq\left\{a_{3}, a_{5}, a_{7}\right\} .
\end{aligned}
$$

Suppose not. Then there exist two vertices $v_{1} \in N_{D}\left(x_{1}\right) \cap N_{D}^{+2}\left(x_{2}\right)-\left\{a_{2}, a_{4}, a_{6}\right\}$ and $v_{2} \in N_{D}\left(y_{1}\right) \cap N_{D}^{+2}\left(y_{2}\right)-\left\{a_{3}, a_{5}, a_{7}\right\}$. By symmetry, we may assume that $v_{1} \in$ $D\left(v_{2}, a_{1}\right)$. If $v_{2}=v_{1}^{-}$, then by replacing $v_{1}^{-} v_{1}$ with $v_{1}^{-} y_{1} x_{1} v_{1}$ in $D$, we get a $C_{2 k+2}^{2}$ in $G$, and this contradiction implies that $v_{2} \neq v_{1}^{-}$. So $v_{2} \in D\left(v_{2}^{-2}, v_{1}^{-3}\right] \subseteq D\left(a_{7}, v_{1}^{-3}\right]$. It follows that

$$
\overline{a_{1} a_{2} \ldots a_{6}} a_{7} \vec{D} v_{2}^{-2} y_{2} x_{2} v_{1}^{-2} \overleftarrow{D} v_{2} y_{1} x_{1} v_{1} \vec{D} a_{1}
$$

is a $C_{2 k+2}^{2}$ in $G$, a contradiction. So (5.2) holds. 
By symmetry, we may assume that

$$
N_{D}\left(x_{1}\right) \cap N_{D}^{+2}\left(x_{2}\right) \subseteq\left\{a_{2}, a_{4}, a_{6}\right\} .
$$

Since $G$ contains no $C_{2 k+2}^{2}$, clearly we have

$$
N_{D}\left(x_{1}\right) \cap N_{D}^{+}\left(y_{1}\right) \subseteq\left\{a_{2}, a_{6}\right\} .
$$

Moreover,

$$
\left|N_{D}\left(y_{1}\right)^{+} \cap N_{D}^{+2}\left(x_{2}\right)-\left\{a_{4}, a_{6}\right\}\right| \leq 1,
$$

for otherwise, let $\left\{u_{1}, u_{2}\right\} \subseteq N_{D}\left(y_{1}\right) \cap N_{D}^{+}\left(x_{2}\right)$ such that $\left\{u_{1}, u_{2}\right\} \cap\left\{a_{3}, a_{5}\right\}=\emptyset$, where $u_{2} \in D\left(u_{1}, a_{1}\right]$. Then

$$
\overline{a_{1} a_{2} \ldots a_{6}} \vec{D} u_{1}^{-} x_{2} u_{2}^{-} \overleftarrow{D} u_{1} y_{1} u_{2} \vec{D} a_{1}
$$

is a $C_{2 k+2}^{2}$ in $G$, a contradiction. Let $z$ be the vertex in $N_{D}\left(y_{1}\right)^{+} \cap N_{D}^{+2}\left(x_{2}\right)-$ $\left\{a_{4}, a_{6}\right\}$, if any. Then each of $a_{2}$ and $a_{4}$ is contained in at most two of the sets in $\left\{N_{D}\left(x_{1}\right), N_{D}\left(y_{1}\right)^{+}, N_{D}^{+2}\left(x_{2}\right)-\{z\}\right\}$. Consequently, $\left|N_{D}\left(x_{1}\right)\right|+\left|N_{D}^{+}\left(y_{1}\right)\right|+\left(\left|N_{D}^{+2}\left(x_{2}\right)\right|-\right.$ $1) \leq\left|V_{2}(D)-\left\{a_{2}, a_{4}, a_{6}\right\}\right|+7=k+4$. By Lemma 2.8 , we have $\left|N_{D}\left(y_{1}\right)\right|=\mid N\left(y_{1}\right)-$ $\left\{x_{1}\right\} \mid \geq n / 3$, and the same holds for $\left|N_{D}\left(x_{i}\right)\right|$ for $i=1,2$. Thus $3(n / 3)-1 \leq k+4$ and hence $k \geq n-5$, contradicting the hypothesis. So Claim 3 is established.

Now let $D$ be a $C_{2 k}^{2}$ in $G$ such that the number of components of $G-D$ is as small as possible. Recall that $4 \leq k \leq n-7$. By Claims 2 and $3, G-D$ contains two isolated vertices $a \in V_{1}-V(D)$ and $b \in V_{2}-V(D)$. From Lemma 4.3 (with $m=2$ ), we see that $G$ contains a $C_{2 k+2}^{2}$. This contradiction completes the proof of our lemma.

The objective of step 3 is to show that every $C_{2 k}$, with $n-6 \leq k \leq n-1$, can be extended to a $C_{2 k+2}$ in $G$.

Lemma 5.2. Let $G=\left(V_{1}, V_{2}, E\right)$ be a balanced bipartite graph with $2 n$ vertices and with a $C_{2 k}$. If $B(G)>3 / 2, n \geq 139$, and $n-6 \leq k \leq n-1$, then $G$ contains a $C_{2 k+2}$.

Proof. Assume on the contrary that $G$ contains no $C_{2 k+2}$. Let $C$ be a $C_{2 k}$ in $G$ such that the number of components of $G-C$ is as small as possible. Let us make some simple observations about $G-C$.

Claim 1. $G-C$ contains no path of length 3 .

Suppose the contrary: $x_{0} x_{1} x_{2} x_{3}$ is a path in $G-C$. By symmetry, we may assume that $x_{0} \in V_{2}$. Since $G$ contains no $C_{2 k+2}$, we deduce that $N_{C}\left(x_{0}\right), N_{C}^{+}\left(x_{1}\right), N_{C}^{+2}\left(x_{2}\right)$, and $N_{C}^{+3}\left(x_{3}\right)$ are disjoint subsets of $V_{1}(C)$. Hence

$$
\left|N_{C}\left(x_{0}\right)\right|+\left|N_{C}^{+}\left(x_{1}\right)\right|+\left|N_{C}^{+2}\left(x_{2}\right)\right|+\left|N_{C}^{+3}\left(x_{3}\right)\right| \leq k .
$$

By Lemma 2.8, we have $\left|N_{C}\left(x_{i}\right)\right| \geq d\left(x_{i}\right)-(n-k) \geq(n+3) / 3-(n-k)=(3 k-2 n+3) / 3$ for $0 \leq i \leq 3$. It follows that $4(3 k-2 n+3) / 3 \leq k$, so $8 n \geq 9 k+12 \geq 9(n-6)+12$ and hence $n \leq 42$, contradicting the hypothesis. Thus Claim 1 is justified.

Claim 2. Each component of $G-C$ contains at most two vertices.

Otherwise, some component $H$ of $G-C$ has at least three vertices. By Claim 1, $H$ contains no path of length 3 . Hence at least one of $V_{1}(H)$ and $V_{2}(H)$ contains only one vertex. Symmetry allows us to assume that $V_{2}(H)=\{u\}$. Then all vertices in $V_{1}(H)$ are adjacent to $u$. Let $v$ be a vertex in $V_{1}(H)$ and set $X:=V_{1}(H)-\{v\}$. Since 
$G$ contains no $C_{2 k+2}$, we see that $N_{C}^{+}(u), N_{C}(v)$, and $N_{C}^{+2}(X)$ are disjoint subsets of $V_{2}(C)$. So $\left|N_{C}^{+}(u)\right|+\left|N_{C}(v)\right|+\left|N_{C}^{+2}(X)\right| \leq k$ and hence

$$
(d(u)-r)+(d(v)-1)+(|N(X)|-1) \leq k,
$$

where $r:=\left|V_{1}(H)\right|$. By Lemma 2.8 , we have $|N(X)| \geq(n+2|X|+1) / 3=(n+2 r-1) / 3$ and $\min \{d(u), d(v)\} \geq(n+3) / 3$. This together with (5.6) implies $3 n \leq 3 k+r+1 \leq$ $n+2 k+1$, so $k>n-1$ and hence $k=n$, and this contradiction justifies Claim 2 .

Claim 3. $G-C$ contains at most one isolated edge.

Assume on the contrary that $x_{1} y_{1}$ and $x_{2} y_{2}$ are two isolated edges of $G-C$ with $\left\{x_{1}, x_{2}\right\} \subseteq V_{1}$ and $\left\{y_{1}, y_{2}\right\} \subseteq V_{2}$. Then $k \leq n-2$. Since $G$ contains no $C_{2 k+2}$, we have $N_{C}\left(x_{i}\right) \cap N_{C}^{+}\left(y_{i}\right)=\emptyset$ for $i=1,2$. It is easy to see that at least one of $N_{C}\left(x_{1}\right) \cap N_{C}^{+2}\left(x_{2}\right)$ and $N_{C}\left(y_{1}\right) \cap N_{C}^{+2}\left(y_{2}\right)$ is empty, for otherwise $G$ would contain a $C_{2 k+2}$, a contradiction. Symmetry allows us to assume that $N_{C}\left(x_{1}\right) \cap N_{C}^{+2}\left(x_{2}\right)=$ $\emptyset$. Then $k \geq\left|N_{C}\left(x_{1}\right) \cup N_{C}^{+2}\left(x_{2}\right) \cup N_{C}^{+}\left(y_{1}\right)\right|=\left|N_{C}\left(x_{1}\right)\right|+\left|N_{C}^{+2}\left(x_{2}\right) \cup N_{C}^{+}\left(y_{1}\right)\right|=$ $\left|N_{C}\left(x_{1}\right)\right|+\left|N_{C}^{+2}\left(x_{2}\right)\right|+\left|N_{C}^{+}\left(y_{1}\right)\right|-\left|N_{C}^{+2}\left(x_{2}\right) \cap N_{C}^{+}\left(y_{1}\right)\right|$. By Lemma 2.8, each of $\left|N_{C}\left(x_{1}\right)\right|,\left|N_{C}^{+}\left(y_{1}\right)\right|$, and $\left|N_{C}^{+2}\left(x_{2}\right)\right|$ is at least $n / 3$. Hence $\left|N_{C}^{+2}\left(x_{2}\right) \cap N_{C}^{+}\left(y_{1}\right)\right| \geq$ $n-k \geq 2$, which again implies the existence of $C_{2 k+2}$ in $G$. This contradiction establishes Claim 3.

Claim 4. $G-C$ contains no isolated vertex.

Otherwise, by Claim 2, there exist $a \in V_{1}-V(C)$ and $b \in V_{2}-V(C)$ such that both of them are isolated vertices in $G-C$ (as $G$ is balanced). From Lemma 4.3 (with $m=0$ ), it follows instantly that $G$ contains a $C_{2 k+2}$, and this contradiction proves Claim 4.

From Claims 1-4, we deduce that $G-C$ contains only two vertices, say, $a$ and $b$, with $a \in V_{1}$ and $b \in V_{2}$, and that $a b \in E$. This in turn implies that $C$ is a longest cycle in $G$. Thus Lemma 4.1 is applicable to the triple $(C ; a, b)$. For each $i \geq 1$ and $v \in\{a, b\}$, let $X_{i}(v)$ and $Y_{i}(v)$ be as defined in this lemma. By definition, (4.1) and Lemma 4.1(ii), $Y_{i}(a) \subseteq V_{1}(C)-X_{1}(b)$. Hence $Y_{i}(a) \cup\{a\}$ is a proper subset of $V_{1}$ for all $i \geq 0$. Similarly, $Y_{i}(b) \cup\{b\}$ is a proper subset of $V_{2}$ for all $i \geq 0$. Therefore, for $i \geq 1$ and $v \in\{a, b\}$, Lemma 2.8 applies to $S=Y_{i-1}(v) \cup\{v\}$. As each of $a$ and $b$ has exactly one neighbor outside $C$, we have

$$
\left|X_{i}(v)\right| \geq \frac{n+2\left(\left|Y_{i-1}(v)\right|+1\right)+1}{3}-1=\frac{n+2\left|Y_{i-1}(v)\right|}{3} .
$$

Thus $3\left|X_{i}(v)\right| \geq n+2\left|Y_{i-1}(v)\right|$. By (4.1) and Lemma 4.1(iv), both $X_{i}(a)^{+}$and $X_{i}(a)^{-}$ are subsets of $V_{1}(C)-X_{i}(b)$. So $\left|X_{i}(a)^{+} \cap X_{i}(a)^{-}\right|=\left|X_{i}(a)^{+}\right|+\left|X_{i}(a)^{-}\right|-\mid X_{i}(a)^{+} \cup$ $X_{i}(a)^{-}|\geq 2| X_{i}(a) \mid-\left(k-\left|X_{i}(b)\right|\right)$. As $X_{i}(a)^{+} \cap X_{i}(a)^{-} \subseteq Y_{i}(a)$, we have

$$
\left|Y_{i}(a)\right| \geq 2\left|X_{i}(a)\right|-\left(k-\left|X_{i}(b)\right|\right) .
$$

Similarly, $\left|Y_{i}(b)\right| \geq 2\left|X_{i}(b)\right|-\left(k-\left|X_{i}(a)\right|\right)$. Adding these two inequalities yields

$$
\left|Y_{i}(a)\right|+\left|Y_{i}(b)\right| \geq 3\left|X_{i}(a)\right|+3\left|X_{i}(b)\right|-2 k .
$$

Hence

$$
\begin{aligned}
\left|Y_{i}(a)\right|+\left|Y_{i}(b)\right| & \geq\left(2 n+2\left|Y_{i-1}(a)\right|+2\left|Y_{i-1}(b)\right|\right)-2 k \\
& =2\left(\left|Y_{i-1}(a)\right|+\left|Y_{i-1}(b)\right|\right)+2(n-k) \\
& \geq 2\left(\left|Y_{i-1}(a)\right|+\left|Y_{i-1}(b)\right|\right)+2
\end{aligned}
$$

Copyright $@$ by SIAM. Unauthorized reproduction of this article is prohibited. 
which implies

$$
\left|Y_{i}(a)\right|+\left|Y_{i}(b)\right|+2 \geq 2\left(\left|Y_{i-1}(a)\right|+\left|Y_{i-1}(b)\right|+2\right) .
$$

Therefore, $\left|Y_{i}(a)\right|+\left|Y_{i}(b)\right|+2 \geq 2^{i+1} \rightarrow \infty$ as $i \rightarrow \infty$. This contradiction completes the proof of our lemma.

Now we are ready to establish the main result of this paper.

Proof of Theorem 1.3. By Lemmas 3.2-3.4, $G$ contains a $C_{4}$, a $C_{6}^{1}$, and at least one of $C_{8}^{2}, C_{10}^{2}$, and $C_{12}^{2}$. By Lemma 5.1, if $G$ contains a $C_{2 k}^{2}$ for any $k$ with $4 \leq$ $k \leq n-7$, then $G$ contains at least one of $C_{2 k+2}^{2}, C_{2 k+4}^{2}$, and $C_{2 k+6}^{2}$. Recall that every $C_{2 t}^{2}$ with $t \geq 4$ contains cycles $C_{2 t}, C_{2 t-2}$, and $C_{2 t-4}$ simultaneously. From all these observations, we conclude that $G$ contains a cycle $C_{2 k}$ for every $k$ with $2 \leq k \leq n-6$. This together with Lemma 5.2 implies that $G$ contains a $C_{2 k}$ for every $k$ with $2 \leq k \leq n$. Therefore $G$ is bipancyclic.

6. Proof of Lemma 4.2. As stated before, Lemma 4.1 aims to deal with a longest cycle under certain restrictions, while Lemma 4.2 is intended for a ladder (not necessarily a longest one) under some other restrictions. Nevertheless, the basic ideas underlying their proofs are essentially similar, and their origin can be traced back to Woodall [8].

The proof of Lemma 4.2 is based on the four claims $A(i, j), B(i, j), B^{*}(i, j)$, and $C(i)$ for all natural numbers $i$ and $j$.

Claim $A(i, j)$. There do not exist two disjoint paths $P_{i j}=u_{1} u_{2} \ldots u_{f}$ and $Q_{i j}=u_{f+1} u_{f+2} \ldots u_{g}$ with the following properties:

(P1) $a_{1} \vec{D} a_{2 m+2}$ is a subpath of either $P_{i j}$ or $Q_{i j}$ when $m \geq 1$;

(P2) $\left\{u_{1}, u_{f+1}\right\} \subseteq X_{i}(a)$ and $\left\{u_{f}, u_{g}\right\} \subseteq X_{j}(b)$;

(P3) if $u_{s} \in Y_{h}(a)$ for some $h<i$ and $s \notin\{f, g\}$, then $\left\{u_{s-1}, u_{s+1}\right\} \subseteq X_{h}(a)$;

(P4) if $u_{s} \in Y_{h}(b)$ for some $h<j$ and $s \notin\{1, f+1\}$, then $\left\{u_{s-1}, u_{s+1}\right\} \subseteq X_{h}(b)$; and

(P5) either $V\left(P_{i j}\right) \cup V\left(Q_{i j}\right)=V(D)$ or $V\left(P_{i j}\right) \cup V\left(Q_{i j}\right)=V(D)-\left\{a_{0}, b_{0}\right\}$ for some $a_{0} \in V_{1}(D)-Y_{i-1}(a)$ and $b_{0} \in V_{2}(D)-Y_{j-1}(b)$ such that $a_{0} b_{0} \in E(G)$.

Claim $B(i, j)$. There does not exist a path $R_{i j}=u_{1} u_{2} \ldots u_{f}$ with the following properties:

(R1) $a_{1} \vec{D} a_{2 m+2}$ is a subpath of $R_{i j}$ when $m \geq 1$;

(R2) $\left\{u_{1}, u_{f}\right\} \subseteq X_{i}(a)$;

(R3) if $u_{s} \in Y_{h}(a)$ for some $h<i$, then $\left\{u_{s-1}, u_{s+1}\right\} \subseteq X_{h}(a)$;

(R4) if $u_{s} \in Y_{h}(b)$ for some $h<j$ and $s \notin\{1, f\}$, then $\left\{u_{s-1}, u_{s+1}\right\} \subseteq X_{h}(b)$; and

(R5) $V\left(R_{i j}\right)=V(D)-\left\{a_{0}\right\}$ for some $a_{0} \in X_{j}(b)$.

Claim $B^{*}(i, j)$. There does not exist a path $R_{i j}^{*}=u_{1} u_{2} \ldots u_{f}$ with the following properties:

(r1) $a_{1} \vec{D} a_{2 m+2}$ is a subpath of $R_{i j}^{*}$ when $m \geq 1$;

(r2) $\left\{u_{1}, u_{f}\right\} \subseteq X_{j}(b)$;

(r3) if $u_{s} \in Y_{h}(b)$ for some $h<j$, then $\left\{u_{s-1}, u_{s+1}\right\} \subseteq X_{h}(b)$;

(r4) if $u_{s} \in Y_{h}(a)$ for some $h<i$ and $s \notin\{1, f\}$, then $\left\{u_{s-1}, u_{s+1}\right\} \subseteq X_{h}(a)$; and

(r5) $V\left(R_{i j}^{*}\right)=V(D)-\left\{b_{0}\right\}$ for some $b_{0} \in X_{i}(a)$.

Claim $C(i)$. For each $v \in\{a, b\}$, there does not exist a path $T_{i}=u_{1} u_{2} \ldots u_{f}$ with the following properties:

(T1) $a_{1} \vec{D} a_{2 m+2}$ is a subpath of $T_{i}$ when $m \geq 1$;

(T2) $\left\{u_{1}, u_{f}\right\} \subseteq X_{i}(v)$;

(T3) if $u_{s} \in Y_{h}(v)$ for some $h<i$ and $s \notin\{1, f\}$, then $\left\{u_{s-1}, u_{s+1}\right\} \subseteq X_{h}(v)$; and 
(T4) $V\left(T_{i}\right)=V(D)-\left\{v_{0}\right\}$ for some $v_{0} \in V(D)-Y_{i-1}(v)$ with $N\left(v_{0}\right) \nsubseteq V(D) \cup\{v\}$. Observe that if $m \geq 1$, then

$$
Y_{j}(v) \cap D\left[a_{1}, a_{2 m+2}\right]=\emptyset \text { for each } v \in\{a, b\} \text { and } j \geq 1,
$$

because $X_{j}(v) \cap D\left[a_{2}, a_{2 m+1}\right]=\emptyset$. We shall repeatedly use this simple observation in the subsequent proofs.

Proof of Lemma 4.2. (assuming Claims $A(i, j), B(i, j), B^{*}(i, j)$, and $C(i)$ for all $i$ and $j$ ).

(i) Suppose the contrary: $N\left(Y_{v}\right)$ is not a subset of $X_{v} \cup D\left(a_{1}, a_{2 m+2}\right)$ for $v=a$ or $b$. By definition, we have $N\left(Y_{i}(v)\right) \nsubseteq X_{i+1}(v) \cup D\left(a_{1}, a_{2 m+2}\right)$ for some $i \geq 1$. So $N\left(Y_{i}(v)\right) \nsubseteq V(D)$ and hence $N\left(v_{0}\right) \notin V(D)$ for some $v_{0} \in Y_{i}(v)$. In view of (6.1), we obtain $v_{0} \notin D\left[a_{1}, a_{2 m+2}\right]$ when $m \geq 1$. Note that $Y_{v}$ and $v$ are both contained in $V_{j}$ for $j=1$ or 2 , so $N\left(v_{0}\right) \nsubseteq \unrhd V(D) \cup\{v\}$. As $Y_{0}(v)=\emptyset$, there exists a subscript $h$ with $1 \leq h \leq i$ such that $v_{0} \in Y_{h}(v)-Y_{h-1}(v)$. Thus $\left\{v_{0}^{-}, v_{0}^{+}\right\} \subseteq X_{h}(v)$, and $v_{0} \in V(D)-Y_{h-1}(v)$. Setting $T_{h}:=v_{0}^{+} \vec{D} v_{0}^{-}$, we see that conditions (T1)-(T4) (with $h$ in place of $i$ ) are all satisfied by $T_{h}$, and hence Claim $C(h)$ is violated. This contradiction implies that $N\left(Y_{v}\right) \subseteq$ $X_{v} \cup D\left(a_{1}, a_{2 m+2}\right)$ for $v=a$ and $b$.

(ii) Suppose $u \in X_{j}(b) \cap Y_{i}(a)$ for some subscripts $i$ and $j$. Then $\left\{u^{-}, u^{+}\right\} \subseteq$ $X_{i}(a)$. By (6.1), $u \notin D\left[a_{1}, a_{2 m+2}\right]$ if $m \geq 1$. Setting $a_{0}:=u$ and $R_{i j}:=$ $u^{+} \vec{D} u^{-}$, we see that conditions (R1)-(R5) are all satisfied by $R_{i j}$, so Claim $B(i, j)$ is violated. Hence $X_{j}(b) \cap Y_{i}(a)=\emptyset$ for all subscripts $i$ and $j$, which implies that $X_{b} \cap Y_{a}=\emptyset$. Similarly, from Claim $B^{*}(i, j)$ we can deduce that $X_{i}(a) \cap Y_{j}(b)=\emptyset$ for all subscripts $i$ and $j$, and hence $X_{a} \cap Y_{b}=\emptyset$.

(iii) Suppose $u$ and $v$ are two distinct vertices in $X_{a}^{+} \cap X_{b}$. Then there exist subscripts $i$ and $j$ such that $\left\{u^{-}, v^{-}\right\} \subseteq X_{i}(a)$ and $\{u, v\} \subseteq X_{j}(b)$. As none of $u^{-}, u, v^{-}$, and $v$ is contained in $D\left[a_{2}, a_{2 m+1}\right]$ when $m \geq 1$, either $D\left[a_{1}, a_{2 m+2}\right] \subseteq D\left[u, v^{-}\right]$or $D\left[a_{1}, a_{2 m+2}\right] \subseteq D\left[v, u^{-}\right]$. Setting $P_{i j}:=u^{-} \overleftarrow{D} v$ and $Q_{i j}:=v^{-} \overleftarrow{D} u$, we see that conditions (P1)-(P5) are all satisfied by $P_{i j}$ and $Q_{i j}$, and hence Claim $A(i, j)$ is violated. This contradiction implies that $\left|X_{a}^{+} \cap X_{b}\right| \leq 1$. Similarly, we have $\left|X_{a}^{-} \cap X_{b}\right| \leq 1$. So the lemma is established.

From the preceding proof, we conclude that

(Z1) Claim $A(i, j)$ implies that $\left|X_{i}(a)^{+} \cap X_{j}(b)\right| \leq 1$ and $\left|X_{i}(a)^{-} \cap X_{j}(b)\right| \leq 1$;

(Z2) Claim $B(i, j)$ implies that $X_{j}(b) \cap Y_{i}(a)=\emptyset$;

(Z3) Claim $B^{*}(i, j)$ implies that $X_{i}(a) \cap Y_{j}(b)=\emptyset$; and

(Z4) Claims $C(h)$, for all $h$ with $1 \leq h \leq i$, imply that $N\left(Y_{i}(v)\right) \subseteq X_{i+1}(v) \cup$ $D\left(a_{1}, a_{2 m+2}\right)$ for $v \in\{a, b\}$.

We shall appeal to these observations in the following inductive proof of the above claims for all possible subscripts.

Proof of Claims $A(1,1), B(1,1), B^{*}(1,1)$, and $C(1)$. Suppose such paths $P_{11}$ and $Q_{11}$ exist. Then $a$ is adjacent to $u_{1}$ and $u_{f+1}$, and $b$ is adjacent to $u_{f}$ and $u_{g}$. If $V\left(P_{11}\right) \cup V\left(Q_{11}\right)=V(D)$, then we can obtain a $C_{2 k+2}^{m}$ from $D$ by adding $a$ and $b$, a contradiction. If $V\left(P_{11}\right) \cup V\left(Q_{11}\right)=V(D)-\left\{a_{0}, b_{0}\right\}$ for some $a_{0} \in V_{1}(D)-Y_{0}(a)$ and $b_{0} \in V_{2}(D)-Y_{0}(b)$ such that $a_{0} b_{0} \in E(G)$. Then we can get another $C_{2 k}^{m}$, denoted by $D^{\prime}$, on the vertex set $\left(V(D)-\left\{a_{0}, b_{0}\right\}\right) \cup\{a, b\}$ such that $G-D^{\prime}$ has at least one component fewer than $G-D$, because both $a$ and $b$ are isolated vertices in $G-D$, while $a_{0} b_{0} \in E(G)$. This contradiction justifies Claim $A(1,1)$. 
Suppose such a path $R_{11}$ exists. Then $a$ is adjacent to $u_{1}$ and $u_{f}$, and $V\left(R_{11}\right)=$ $V(D)-\left\{a_{0}\right\}$ for some $a_{0} \in X_{1}(b)$ (so $a_{0} b \in E(G)$ ). Hence we can obtain another $C_{2 k}^{m}$, denoted by $D^{\prime}$, on the vertex set $\left(V(D)-\left\{a_{0}\right\}\right) \cup\{a\}$ such that $G-D^{\prime}$ has at least one component fewer than $G-D$, because both $a$ and $b$ are isolated vertices in $G-D$, while $a_{0} b \in E(G)$. This contradiction justifies Claim $B(1,1)$. Similarly, Claim $B^{*}(1,1)$ also holds.

Suppose such a path $T_{1}$ exists. Then $v$ is adjacent to $u_{1}$ and $u_{f}$, and $V\left(T_{1}\right)=$ $V(D)-\left\{v_{0}\right\}$ for some $v_{0} \in V(D)$ with $N\left(v_{0}\right) \nsubseteq V(D) \cup\{v\}$. Hence we can obtain another $C_{2 k}^{m}$, denoted by $D^{\prime}$, on the vertex set $\left(V(D)-\left\{v_{0}\right\}\right) \cup\{v\}$ such that $G-D^{\prime}$ has at least one component fewer than $G-D$, because $v$ is an isolated vertex in $G-D$ while $N\left(v_{0}\right) \nsubseteq V(D) \cup\{v\}=V\left(D^{\prime}\right) \cup\left\{v_{0}\right\}$. This contradiction justifies Claim $C(1)$.

Proof of Claims $A(i, j), B(i, j)$, and $B^{*}(i, j)$ for $i+j>2$. We proceed by induction on $i+j$. Suppose $i+j>2$ and $A\left(i_{0}, j_{0}\right), B\left(i_{0}, j_{0}\right), B^{*}\left(i_{0}, j_{0}\right)$ hold for all subscripts $i_{0}$ and $j_{0}$ with $i_{0}+j_{0}<i+j$.

(1) To prove $A(i, j)$, suppose on the contrary that such paths $P_{i j}$ and $Q_{i j}$ exist. By symmetry, we may assume that $i \geq j$ (so $i>1$ ). Let us distinguish among three cases.

Case A1. $\left\{u_{1}, u_{f+1}\right\} \subseteq X_{i-1}(a)$.

In this case let $P_{i-1, j}:=P_{i j}$ and $Q_{i-1, j}:=Q_{i j}$. Then the existence of such two paths contradicts Claim $A(i-1, j)$.

Case A2. Precisely one of $u_{1}$ and $u_{f+1}$ is in $X_{i-1}(a)$.

In this case symmetry allows us to assume that $u_{1} \in X_{i-1}(a)$, while $u_{f+1} \notin$ $X_{i-1}(a)$. Then $u_{f+1}$ is adjacent to some $y \in Y_{i-1}(a)-Y_{i-2}(a)$. If $y \notin V\left(P_{i j} \cup Q_{i j}\right)$, then, by (P5), we have $V\left(P_{i j} \cup Q_{i j}\right)=V(D)-\left\{a_{0}, b_{0}\right\}$ and $y \in\left\{a_{0}, b_{0}\right\}$, where $a_{0} \in V_{1}(D)-Y_{i-1}(a)$ and $b_{0} \in V_{2}(D)-Y_{j-1}(b)$. It follows that $y=a_{0} \notin Y_{i-1}(a)$, a contradiction. Hence $y=u_{s}$ for some $s$ with $1 \leq s \leq g$. By (6.1), $u_{s} \notin D\left[a_{1}, a_{2 m+2}\right]$ when $m \geq 1$. By Claim $B(i-1, j)$ and (Z2), $X_{j}(b) \cap Y_{i-1}(a)=\emptyset$, so $s \notin\{f, g\}$. As $\left\{u_{1}, u_{f+1}\right\} \subseteq X_{i}(a)$, we see that $u_{1} \in V_{2}$ and $u_{s} \in N\left(u_{f+1}\right) \subseteq V_{1}$, and hence $s \notin\{1, f+1\}$. Consequently, either $1<s<f$ or $f+1<s<g$. By (P3), we have $\left\{u_{s-1}, u_{s+1}\right\} \subseteq X_{i-1}(a)$. Set

$$
\begin{aligned}
P_{i-1, j} & := \begin{cases}u_{1} \overrightarrow{P_{i j}} u_{s} u_{f+1} \overrightarrow{Q_{i j}} u_{g} & \text { if } 1<s<f, \\
P_{i j} & \text { if } f+1<s<g,\end{cases} \\
Q_{i-1, j}: & = \begin{cases}u_{s+1} \overrightarrow{P_{i j}} u_{f} & \text { if } 1<s<f, \\
u_{s-1} \vec{Q}_{i j} u_{f+1} u_{s} \overrightarrow{Q_{i j}} u_{g} & \text { if } f+1<s<g .\end{cases}
\end{aligned}
$$

Let us show that (P1)-(P5) (with $i-1$ in place of $i$ ) are all satisfied by $P_{i-1, j}$ and $Q_{i-1, j}$. Suppose $1<s<f$. Then the details of the proof are given below.

(P1) As $u_{s} \notin D\left[a_{1}, a_{2 m+2}\right]$, it is clear that $a_{1} \vec{D} a_{2 m+2}$ remains a subpath of either $P_{i-1, j}$ or $Q_{i-1, j}$ when $m \geq 1$.

(P2) By assumption, $u_{1} \in X_{i-1}(a)$ and $\left\{u_{f}, u_{g}\right\} \subseteq X_{j}(b)$. As remarked above, $u_{s-1} \in X_{i-1}(a)$.

(P3) Since $P_{i j}$ and $Q_{i j}$ satisfy (P3), the only possible vertex on $P_{i-1, j} \cup Q_{i-1, j}$ that can violate (P3) is $u_{s}$. However, since $u_{s} \notin Y_{i-2}(a)$, we have $u_{s} \notin Y_{h}(a)$ for all $h<i-1$ by (4.1).

(P4) Since $P_{i j}$ and $Q_{i j}$ satisfy (P4), the only possible vertex on $P_{i-1, j} \cup Q_{i-1, j}$ that can violate $(\mathrm{P} 4)$ is $u_{f+1}$. However, since Claim $B(i-1, j)$ implies $X_{j}(b) \cap$ $Y_{i-1}(a)=\emptyset($ recall $(\mathrm{Z} 2))$, we have $u_{s} \notin X_{j}(b)$. This together with $u_{f+1} u_{s} \in$ 
$E(G)$ and $u_{s} \in V(D)-D\left(a_{1}, a_{2 m+2}\right)$ implies $u_{f+1} \notin Y_{j-1}(b)$, and hence $u_{f+1} \notin Y_{h}(b)$ for all $h<j$ by $(4.1)$.

(P5) This follows from the fact that $V\left(P_{i j} \cup Q_{i j}\right)=V\left(P_{i-1, j} \cup Q_{i-1, j}\right)$ and that $P_{i j}$ and $Q_{i j}$ satisfy (P5). Also, if $P_{i j}$ and $Q_{i j}$ miss $a_{0} \in V_{1}(D)-Y_{i-1}(a)$, then we have $a_{0} \in V_{1}(D)-Y_{i-2}(a)$ as well by $(4.1)$.

The proof goes along the same line when $f+1<s<g$.

Case A3. $\left\{u_{1}, u_{f+1}\right\} \cap X_{i-1}(a)=\emptyset$.

As in Case A2, we can now deduce that $u_{1}$ is adjacent to some $u_{r} \in Y_{i-1}(a)-$ $Y_{i-2}(a)$, and $u_{f+1}$ is adjacent to some $u_{s} \in Y_{i-1}(a)-Y_{i-2}(a)$, where $2 \leq r, s \leq g-1$ and $\{r, s\} \cap\{f, f+1\}=\emptyset$. By (P3), we have $\left\{u_{r-1}, u_{r+1}, u_{s-1}, u_{s+1}\right\} \subseteq X_{i-1}(a)$. By (6.1), we obtain $\left\{u_{r}, u_{s}\right\} \cap D\left[a_{1}, a_{2 m+2}\right]=\emptyset$ when $m \geq 1$. Symmetry allows us to assume that $u_{r} \in P_{i j}$ whenever $u_{r}=u_{s}$ and $r<s$ whenever $u_{r}$ and $u_{s}$ are two distinct vertices both on $P_{i j}$ or both on $Q_{i j}$. Thus there are four possibilities for $r$ and $s$ altogether: (i) $1<r<f<f+1<s<g$; (ii) $1<s<f<f+1<r<g$; (iii) $1<r \leq s<f$; or (iv) $f+1<r<s<g$. Set

$$
\begin{aligned}
& P_{i-1, j}:= \begin{cases}u_{r-1} \overleftarrow{P_{i j}} u_{1} u_{r} \overrightarrow{P_{i j}} u_{f} & \text { if } 1<r<f<f+1<s<g, \\
u_{r-1} \overleftarrow{Q_{i j}} u_{f+1} u_{s} \overrightarrow{P_{i j}} u_{f} & \text { if } 1<s<f<f+1<r<g, \\
u_{s+1} \overleftrightarrow{P_{i j}} u_{f} & \text { if } 1<r \leq s<f, \\
u_{s-1} \overleftarrow{Q_{i j}} u_{r} u_{1} \overrightarrow{P_{i j}} u_{f} & \text { if } f+1<r<s<g,\end{cases} \\
& Q_{i-1, j}:= \begin{cases}u_{s-1} \overleftarrow{Q_{i j}} u_{f+1} u_{s} \overrightarrow{Q_{i j}} u_{g} & \text { if } 1<r<f<f+1<s<g, \\
u_{s-1} \overleftarrow{P_{i j}} u_{1} u_{r} \overrightarrow{Q_{i j}} u_{g} & \text { if } 1<s<f<f+1<r<g \\
u_{r-1} \overleftarrow{P}_{i j} u_{1} u_{r} \overrightarrow{P_{i j}} u_{s} u_{f+1} \overrightarrow{Q_{i j}} u_{g} & \text { if } 1<r \leq s<f, \\
u_{r-1} \overleftarrow{Q_{i j}} u_{f+1} u_{s} \overrightarrow{Q_{i j}} u_{g} & \text { if } f+1<r<s<g .\end{cases}
\end{aligned}
$$

Again, it is a routine matter to check that $\left(P_{i-1, j}, Q_{i-1, j}\right)$ satisfies (P1)-(P5) (with $i-1$ in place of $i)$. This contradiction to Claim $A(i-1, j)$ completes the proof for the present case. Therefore Claim $A(i, j)$ is established.

(2) Let us now justify Claims $B(1, j)$ for $j>1$. Assume such a path $R_{1 j}$ exists with corresponding $a_{0} \in X_{j}(b)$. Then $a_{0} \notin X_{j-1}(b)$, for otherwise Claim $B(1, j-1)$ is violated. Hence $a_{0}$ is adjacent to some $u_{r} \in Y_{j-1}(b)-Y_{j-2}(b)$, where $1 \leq r \leq f$. By Claim $B^{*}(1, j-1)$ and (Z3), we have $Y_{j-1}(b) \cap X_{1}(a)=\emptyset$, so $u_{r} \notin X_{1}(a)$. This together with $\left\{u_{1}, u_{f}\right\} \subseteq X_{1}(a)$ implies $1<r<f$. By (R4), $\left\{u_{r-1}, u_{r+1}\right\} \subseteq X_{j-1}(b)$. By (6.1), we have $u_{r} \notin D\left[a_{1}, a_{2 m+2}\right]$ when $m \geq 1$. Let $P_{1, j-1}:=u_{1} u_{2} \ldots u_{r-1}$, $Q_{1, j-1}:=u_{f} u_{f-1} \ldots u_{r+1}$, and $b_{0}:=u_{r}$. Then it is easy to see that (P1)-(P5) (with $(1, j-1)$ in place of $(i, j))$ are all satisfied by $\left(P_{1, j-1}, Q_{1, j-1}\right)$. This contradiction to Claim $A(1, j-1)$ establishes Claim $B(1, j)$ for all $j>1$.

Similarly, we can justify Claim $B^{*}(1, j)$ for all $j>1$.

(3) Next, let us justify Claim $B(i, j)$ for $i>1$. Assume such a path $R_{i j}$ exists with corresponding $a_{0} \in X_{j}(b)$. We consider three cases.

Case B1. $\left\{u_{1}, u_{f}\right\} \subseteq X_{i-1}(a)$.

In this case set $R_{i-1, j}:=R_{i j}$. Then the existence of this path contradicts Claim $B(i-1, j)$.

Case B2. Precisely one of $u_{1}$ and $u_{f}$ is in $X_{i-1}(a)$.

In this case symmetry allows us to assume that $u_{1} \in X_{i-1}(a)$ while $u_{f} \notin X_{i-1}(a)$. Then $u_{f}$ is adjacent to some $y \in Y_{i-1}(a)-Y_{i-2}(a)$. By Claim $B(i-1, j)$ and (Z2), we have $X_{j}(b) \cap Y_{i-1}(a)=\emptyset$, so $y \neq a_{0}$, for otherwise $a_{0} \in X_{j}(b) \cap Y_{i-1}(a)$, a contradiction. In view of (R5), we have $V(D)=V\left(R_{i j}\right) \cup\left\{a_{0}\right\}$, so $y \in V\left(R_{i j}\right)$ and 
hence $y=u_{r}$ for some $r$ with $1 \leq r \leq f$. By (6.1), we get $u_{r} \notin D\left[a_{1}, a_{2 m+2}\right]$ when $m \geq 1$. As $\left\{u_{1}, u_{f}\right\} \subseteq X_{i}(a) \subseteq V_{2}$ and $u_{r} \in N\left(u_{f}\right) \subseteq V_{1}$, we obtain $1<r<f$. It follows from (R3) that $u_{r+1} \in X_{i-1}(a)$. Let $R_{i-1, j}:=u_{1} u_{2} \ldots u_{r} u_{f} u_{f-1} \ldots u_{r+1}$. Then (R1)-(R5) (with $i-1$ in place of $i$ ) are all satisfied by $R_{i-1, j}$; the details of the proof are given below.

(R1) Since $u_{r} \notin D\left[a_{1}, a_{2 m+2}\right]$, it is clear that $a_{1} \vec{D} a_{2 m+2}$ remains a subpath of $R_{i-1, j}$ when $m \geq 1$.

(R2) By assumption, $u_{1} \in X_{i-1}(a)$. As remarked above, $u_{r+1} \in X_{i-1}(a)$.

(R3) Since $R_{i j}$ satisfies (R3), the only possible vertex on $R_{i-1, j}$ that can violate (R3) is $u_{r}$. However, since $u_{r} \notin Y_{i-2}(a)$, we have $u_{r} \notin Y_{h}(a)$ for all $h<i-1$ by (4.1).

(R4) Since $R_{i j}$ satisfies (R4), the only possible vertex on $R_{i-1, j}$ that can violate (R4) is $u_{f}$. However, by Claim $B(i-1, j)$ and (Z2), we have $X_{j}(b) \cap$ $Y_{i-1}(a)=\emptyset$, so $u_{r} \notin X_{j}(b)$. This together with $u_{f} u_{r} \in E(G)$ and $u_{r} \in$ $V(D)-D\left(a_{1}, a_{2 m+2}\right)$ implies $u_{f} \notin Y_{j-1}(b)$, and hence $u_{f} \notin Y_{h}(b)$ for all $h<j$ by $(4.1)$.

(R5) This follows from the fact that $V\left(R_{i j}\right)=V\left(R_{i-1, j}\right)$ and that $R_{i j}$ satisfies (R5).

Therefore the existence of $R_{i-1, j}$ contradicts Claim $B(i-1, j)$.

Case B3. $\left\{u_{1}, u_{f}\right\} \cap X_{i-1}(a)=\emptyset$.

As in Case B2, we can now deduce that $u_{1}$ is adjacent to some $u_{s}$, and $u_{f}$ is adjacent to some $u_{r}$, where $\left\{u_{s}, u_{r}\right\} \subseteq Y_{i-1}(a)-Y_{i-2}(a)$ and $1<s, r<f$. By (R3), we have $\left\{u_{r-1}, u_{r+1}, u_{s-1}, u_{s+1}\right\} \subseteq X_{i-1}(a)$. By (6.1), we obtain $\left\{u_{r}, u_{s}\right\} \cap$ $D\left[a_{1}, a_{2 m+2}\right]=\emptyset$ when $m \geq 1$. Set

$$
R_{i-1, j}:= \begin{cases}u_{s-1} \overleftarrow{R_{i j}} u_{1} u_{s} \overrightarrow{R_{i j}} u_{r} u_{f} \overleftarrow{R_{i j}} u_{r+1} & \text { if } r \geq s \\ u_{r-1} \overleftarrow{R_{i j}} u_{1} u_{s} \overrightarrow{R_{i j}} u_{f} u_{r} \widehat{R_{i j}} u_{s-1} & \text { if } r<s\end{cases}
$$

It is then a routine matter to check that (R1)-(R5) (with $i-1$ in place of $i$ ) are all satisfied by $R_{i-1, j}$. Thus the existence of $R_{i-1, j}$ contradicts Claim $B(i-1, j)$.

Similarly, we can justify Claim $B^{*}(i, j)$ for all $i>1$.

Proof of Claim $C(i)$ for $i>1$. We proceed by induction on $i$. Suppose $i>1$ and $C\left(i_{0}\right)$ holds for all $i_{0}$ with $1 \leq i_{0}<i$. To prove $C(i)$, assume on the contrary that such a path $T_{i}$ exists with corresponding $v_{0} \notin Y_{i-1}(v)$ such that $N\left(v_{0}\right) \nsubseteq V(D) \cup\{v\}$, where $v \in\{a, b\}$. We consider three cases.

Case C1. $\left\{u_{1}, u_{f}\right\} \subseteq X_{i-1}(v)$.

In this case set $T_{i-1}:=T_{i}$. Then the existence of this path contradicts Claim $C(i-1)$.

Case C2. Precisely one of $u_{1}$ and $u_{f}$ is in $X_{i-1}(v)$.

In this case symmetry allows us to assume that $u_{1} \in X_{i-1}(v)$ while $u_{f} \notin X_{i-1}(v)$. Then $u_{f}$ is adjacent to some $y \in Y_{i-1}(v)-Y_{i-2}(v)$. As $v_{0} \notin Y_{i-1}(v)$, we have $y \neq v_{0}$. Using (T4), we see that $y \in V\left(T_{i}\right)$, so $y=u_{r}$ for some $r$ with $1 \leq r \leq f$. In view of (6.1), we obtain $u_{r} \notin D\left[a_{1}, a_{2 m+2}\right]$ when $m \geq 1$. Since both $u_{1}$ and $u_{f}$ are in $V_{i}$ for $i=1$ or 2 , we deduce that $1<r<f$. Using (T3), we get $\left\{u_{r-1}, u_{r+1}\right\} \subseteq X_{i-1}(v)$. Let $T_{i-1}:=u_{1} u_{2} \ldots u_{r} u_{f} u_{f-1} \ldots u_{r+1}$. We can now show that (T1)-(T4) (with $i-1$ in place of $i$ ) are all satisfied by $T_{i-1}$; the details of the proof are given below.

(T1) Since $u_{r} \notin D\left[a_{1}, a_{2 m+2}\right]$, it is clear that $a_{1} \vec{D} a_{2 m+2}$ must remain a subpath of $T_{i-1}$ when $m \geq 1$.

(T2) By assumption, $u_{1} \in X_{i-1}(v)$. As noted above, $u_{r+1} \in X_{i-1}(v)$. 
(T3) Since $T_{i}$ satisfies (T3), the only possible vertex on $T_{i-1}$ that can violate (T3) is $u_{r}$. However, since $u_{r} \notin Y_{i-2}(v)$, we have $u_{r} \notin Y_{h}(v)$ for all $h<i-1$ by (4.1).

(T4) This follows from the fact that $V\left(T_{i}\right)=V\left(T_{i-1}\right)$ and that $T_{i}$ satisfies (T4). As $v_{0} \notin Y_{i-1}(v)$, we have $v_{0} \notin Y_{i-2}(v)$ as well by (4.1).

Hence the existence of $T_{i-1}$ contradicts Claim $C(i-1)$.

Case C3. $\left\{u_{1}, u_{f}\right\} \cap X_{i-1}(v)=\emptyset$.

As in Case C2, we can now deduce that $u_{1}$ is adjacent to some $u_{s}$, and $u_{f}$ is adjacent to some $u_{r}$, where $\left\{u_{s}, u_{r}\right\} \subseteq Y_{i-1}(v)-Y_{i-2}(v)$ and $1<s, r<f$. By (T3), we have $\left\{u_{r-1}, u_{r+1}, u_{s-1}, u_{s+1}\right\} \subseteq X_{i-1}(a)$. By (6.1), we obtain $\left\{u_{r}, u_{s}\right\} \cap$ $D\left[a_{1}, a_{2 m+2}\right]=\emptyset$ when $m \geq 1$. Set

$$
T_{i-1}:= \begin{cases}u_{s-1} \overleftarrow{T_{i}} u_{1} u_{s} \vec{T}_{i} u_{r} u_{f} \overleftarrow{T_{i}} u_{r+1} & \text { if } r \geq s \\ u_{r-1} \overleftarrow{T_{i}} u_{1} u_{s} \vec{T}_{i} u_{f} u_{r} \vec{T}_{i} u_{s-1} & \text { if } r<s\end{cases}
$$

It is then a routine matter to check that (T1)-(T4) (with $i-1$ in place of $i$ ) are all satisfied by $T_{i-1}$. Thus the existence of $T_{i-1}$ contradicts Claim $C(i-1)$.

This completes the proof of all the claims.

\section{REFERENCES}

[1] P. Ash, Dominating Cycles, Hamilton Cycles and Cycles with Many Chords in 2-Connected Graphs, Ph.D. thesis, Goldsmiths' College, London, 1985.

[2] R. HÄGgkvist, Unsolved Problems, in Proceedings of the Fifth Hungarian Colloquium on Combinatorics, 1976.

[3] B. JaCKSON, Hamilton cycles in regular 2-connected graphs, J. Combin. Theory Ser. B, 29 (1980), pp. 27-46.

[4] B. JaCKSON AND H. LI, Hamilton cycles in 2-connected regular bipartite graphs, J. Combin. Theory Ser. B, 62 (1994), pp. 236-258.

[5] I. Reiman, Über ein Problem von K. Zarankiewicz, Acta Math. Acad. Sci. Hungar., 9 (1958), pp. 269-273.

[6] R. SHI, The binding number of a graph and its triangle, Acta Math. Appl. Sinica (English Ser.), 3 (1985), pp. 79-86.

[7] R. SHI, The binding number of a graph and its pancyclicism, Acta Math. Appl. Sinica (English Ser.), 3 (1987), pp. 257-269.

[8] D. R. Woodall, The binding number of a graph and its Anderson number, J. Combin. Theory Ser. B, 15 (1973), pp. 225-255.

Copyright $@$ by SIAM. Unauthorized reproduction of this article is prohibited. 